Hydrol. Earth Syst. Sci. Discuss., doi:10.5194/hess-2017-9, 2017

Manuscript under review for journal Hydrol. Earth Syst. Sci.

Published: 17 January 2017

(c) Author(s) 2017. CC-BY 3.0 License.

\title{
Bayesian joint inference of hydrological and generalized error models with the enforcement of Total Laws
}

\author{
Mario R. Hernández-López ${ }^{1}$, Félix Francés ${ }^{1}$ \\ ${ }^{1}$ Research Institute of Water and Environmental Engineering, Universitat Politècnica de València, Spain \\ Correspondence to: Mario R. (maherlo@hma.upv.es)
}

\begin{abstract}
.
Over the years, the Standard Least Squares (SLS) has been the most commonly adopted criterion for the calibration of hydrological models, despite the fact that they generally do not fulfill the assumptions made by the SLS method: very often errors are autocorrelated, heteroscedastic, biased and/or non-Gaussian. Similarly to recent papers, which suggest more appropriate models for the errors in hydrological modeling, this paper addresses the challenging problem of jointly estimate hydrological and error model parameters (joint inference) in a Bayesian framework, trying to solve some of the problems found in previous related researches. This paper performs a Bayesian joint inference through the application of different inference models, as the known SLS or WLS and the new GL++ and GL++Bias error models. These inferences were carried out on two lumped hydrological models which were forced with daily hydrometeorological data from a basin of the MOPEX project. The main finding of this paper is that a joint inference, to be statistically correct, must take into account the joint probability distribution of the state variable to be predicted and its deviation from the observations (the errors). Consequently, the relationship between the marginal and conditional distributions of this joint distribution must be taken into account in the inference process. This relation is defined by two general statistical expressions called the Total Laws (TLs): the Total Expectation and the Total Variance Laws. Only simple error models, as SLS, do not explicitly need the TLs implementation. An important consequence of the TLs enforcement is the reduction of the degrees of freedom in the inference problem namely, the reduction of the parameter space dimension. This research demonstrates that non-fulfillment of TLs produces incorrect error and hydrological parameter estimates and unreliable predictive distributions. The target of a (joint) inference must be fulfilling the error model hypotheses rather than to achieve the better fitting to the observations. Consequently, for a given hydrological model, the resulting performance of the prediction, the reliability of its predictive uncertainty, as well as the robustness of the parameter estimates, will be exclusively conditioned by the degree in which errors fulfill the error model hypotheses.
\end{abstract}




\section{Introduction}

In hydrologic simulation, many parameters are not directly observable or easily inferred from measured data. For this reason, these parameters must be estimated indirectly by an inverse process (also called calibration) that conditions the parameter estimates and the model response on historically observed input-output data (Pokhrel and Gupta, 2010). Model parameter inferences are based on a likelihood function which quantifies the probability that the observed data were generated by a particular parameter set (Box and Tiao, 1992). Over the years, the Standard Least Squares (SLS) criterion has been the most commonly adopted method for the calibration of the unknown parameters in hydrological modeling, although it has long been known that errors of hydrologic models are generally autocorrelated and heteroscedastic (Sorooshian and Dracup, 1980) not fulfilling the underlying hypotheses to SLS. Also, model inputs and model structural errors are important sources of uncertainty and they are ignored by the SLS scheme. I.e., the SLS parameter estimates are adjusted with a particular calibration data set to partially compensate the input errors (Kavetski et al., 2003; Schoups and Vrugt, 2010) and/or the structural errors (Schoups and Vrugt, 2010) yielding a set of biased parameters.

The present paper is framed as a formal Bayesian aggregated approach to characterize the parameter and total predictive uncertainty, with a direct method for handling the bias, autocorrelation, heteroscedasticity and non-normality of the hydrological model errors. This approach follows to that proposed in Schoups and Vrugt (2010), but in present paper we have introduced significant improvements. Schoups and Vrugt (2010) proposed a formal likelihood function based on a general error model (called GL error model) that allows for model bias and for autocorrelation, non-stationarity, and non-normality of model errors. This formal approach preserved the advantages of being developed from a theoretical basis and having the possibility of checking the assumptions, while at the same time it improved flexibility and reduced the need for unrealistic assumptions about the errors. That study was based on the joint inference of both the hydrological and error model parameters (hereinafter joint inference). The main contribution of that research was the treatment of the error heteroscedasticity and non-normality with a direct method instead of using a transformational one, as it had been previously used in several case studies (Bates and Campbell, 2001; Kuczera, 1983; Reichert and Mieleitner, 2009; Vrugt et al., 2009b). However, according to Schoups and Vrugt (2010), something in the method seemed not to work properly: the analysis performed with heteroscedasticity, autocorrelation, and non-normality error parameters for the second case study (Guadalupe River basin) gave results with "large and meaningless" prediction uncertainty bands. These authors pointed out that the reason was the large inferred value for the autocorrelation coefficient. To avoid this problem, the authors decided to adjust the autocorrelation parameter to an observed sampling value, rather than inferring it jointly and automatically.

Evin et al. (2013) described the challenges of fitting hydrological model parameters jointly with the autocorrelation and heteroscedasticity parameters of error models, and found a solution to the aforementioned problem in Schoups and Vrugt (2010). The key to the problem solution depended on how the autoregressive model was applied. Applying it directly to the errors can produce instability in the computations and, as a result, a poor error model with large predictive uncertainty. This instability could be avoided by applying the autoregressive process to the studentized errors. Thus, the heteroscedasticity model was performed on errors (instead on innovations) and the de-correlation process was applied on the previously variance-stabilized errors.

In a useful and comprehensive research on the comparison of inference methodologies, Evin et al. (2014) detected that problems remained in the joint estimation of both hydrological and error model parameters. They concluded that the joint inference could be non-robust due to multiway interactions between the hydrological parameters (related with the water balance in their case study) and the error model parameters, particularly, heteroscedasticity and autocorrelation error model parameters.

Moreover, several related researches have avoided a full joint inference either without the modeling of the error's autocorrelation (Cheng et al., 2014; Westra et al., 2014) or using a transformational (or indirect) method for the treatment of the error heteroscedasticity (Bates and Campbell, 2001; Cheng et al., 2014; Del Giudice et al., 2013; Kuczera, 1983; Reichert and Mieleitner, 2009; Vrugt et al., 2009b). Even there are authors who combine the Markov Chain Monte Carlo (MCMC) sampling of the hydrological parameters with a maximum likelihood estimation of the error model parameters conditioned on each MCMC hydrological parameter sample (Zheng and Han, 2015; Han and Zheng, 2016), making a sort of pseudo-joint 
Hydrol. Earth Syst. Sci. Discuss., doi:10.5194/hess-2017-9, 2017

inference. However other authors as Scharnagl et al.(2015) tried the joint inference without success, finding similar problems to those exposed in Schoups and Vrugt (2010) or Evin et al. (2013, 2014).

Consequently, the general purpose of our research is to find out the reasons and to overcome the drawbacks identified in the previous studies, defending the reliability of the Bayesian joint inference methodology. This defense is necessary because non-using the joint inference involves a range of problems (Evin et al., 2014): i) the use of a faulty error model when calibrating the hydrological parameters produces biased parameter estimates and an incorrect parameter and predictive uncertainty estimates; ii) the estimation of error model parameters conditioned on a particular estimate of hydrological parameters (assuming that they could be biased) ignores interactions between both sets of parameters, and may produce an incorrect estimation of the predictive uncertainty.

The specific objectives covered by this paper are the following. Firstly, we have improved the formal general likelihood function for parameter and predictive inference of hydrological models developed by Schoups and Vrugt (2010), implementing the previously mentioned recommendation proposed in Evin et al. (2013). Secondly, this paper seeks to shed some light on the causes of the incorrect behavior of the joint inference which has been detected in Schoups and Vrugt (2010), Evin et al. (2013, 2014) and Scharnagl et al. (2015).

Todini (2007) pointed out that “(...) uncertainty (...) is 'conditional' upon the forecasting model prediction”. That idea is the basis to understand that the concept of predictive uncertainty must be linked with the conditional distribution of a predicted variable given its related model prediction namely $p\left(y \mid y_{s}\right)$. Therefore, the existence of that conditional distribution implies also the existence of its joint distribution $p\left(y, y_{s}\right)$, which is the foundation of the general framework proposed in this paper. When we define the conditional distributions through any of its statistical features (i.e. shape, position, etc.), it is important to note that we are defining in parts their parent joint distribution. So, these parts must comply with the necessary conditions/restrictions to conform all together such joint distribution. In particular, two necessary conditions are the Total Variance Law and the Total Expectation Law (for details about these laws see for example Blitzstein and Hwang, 2014), which relate the marginal and conditional distributions of a given joint distribution. This paper enforces both Total Laws to the heteroscedasticity and non-constant bias models, since they are part of the conditional distributions modeling and, hence, they belong to the joint probability density function (hereinafter pdf). The presented case studies will show how not enforcing the Total Laws could be the origin of the previously found problems with the Bayesian joint inference.

The next section presents the generalized error statistical model followed by this research. "General” in the sense that error model permits the possibility for the errors to be autocorrelated, heteroscedastic, biased and/or non-Gaussian. Section 3 deals with the joint Bayesian parameter inference. A new formal generalized likelihood function is presented and the methods for obtaining the posterior of parameters and the predictive distribution are outlined. Section 4 describes the different inference settings and how to apply the Total Laws on them. Section 5 applies the exposed methodology to estimate the parameter and predictive uncertainty, through the joint inference with several error models and two lumped hydrological daily models, using hydrometeorological data from a basin in the Model Parameter Estimation Experiment (MOPEX) data set (Duan et al., 2006). The fulfillment of assumptions in the hypothesized error models, as well as several indicators of performance will be tested. Section 6 discusses the results and section 7 summarizes our findings.

\section{Generalized error statistical model}

\section{$83 \quad 2.1 \quad$ Why do we need a reliable error model?}

84 Let us consider a random variable of hydrological predictive interest to be forecasted, for which the observations are made and which will be called the predictand $y$ (e.g. the streamflow at a catchment outlet $\boldsymbol{q}$, or any other state variable of interest). The predictand $y$ can be sampled at $N$ constant time steps and it is jointly observed and sampled with a set of predictors or model inputs, $\mathbf{X}$ (e.g. precipitation, temperature, etc.). This research will consider $\mathbf{X}$ as deterministic variables, which are able to explain "something” about the predictand's behavior. 
Hydrol. Earth Syst. Sci. Discuss., doi:10.5194/hess-2017-9, 2017

Let us also consider any model output $y_{s}$ (e.g. the simulated streamflow $q_{s}$, or some other observable and simulated state variable) as a random variable; not because a stochastic model is used (which is not our case), but by the fact that we can observe that these simulated variables generally do not match their observations. In this sense, Todini (2007) pointed out that “ (...) a scatter will always be observed in the $q-q_{s}$ plane (...) a representation of the joint sample frequency of $q$ and $q_{s}$ that can be used to estimate its joint probability density". That is to say, there is an inherent uncertainty whose origin is in the model structure soundness, in the observed data errors of predictand and predictors, and ultimately in the inherent unpredictability in deterministic terms of the natural phenomena. Montanari and Koutsoyiannis (2012) concluded that uncertainty is unavoidable in Hydrology so it is impossible to produce a fully deterministic model that would eliminate the uncertainty, and modeling schemes need to explicitly recognize its role.

As stated in Todini (2007), two different objectives can be differentiated in hydrological modeling: parameter estimation and hydrological prediction. Parameter estimation is the procedure for obtaining parameter values with a physical meaning, which help us to understand the nature of the modeled processes. That is to say, the aim of the hydrological parameter estimation is not (it should not be) to get the best fit with the observations, but achieving the most plausible estimates with the best possible error model for the given observed data set. Hydrologists should assume the fact that, given a correct error model (actually a correct likelihood function), the most plausible parameter set can produce simulated results which show a poor fit to the observations, even in the calibration period. In other words, an inference with the correct error model yields the most plausible parameter set, which will not necessarily yield the best fit to the observed data, but will produce the best possible result, taking into account the limitations of the hydrological model and the observational errors. In a classical Frequentist context, the most plausible parameters provide the Maximum Likelihood and in a Bayesian framework are the Maximum a Posteriori (from now MAP).

With regard to the problem of making predictions about some variable of interest, the correct estimation of the hydrological parameters is not necessary, since the predictive uncertainty, $p\left(y \mid y_{s}\right)$, can be obtained through the direct modeling of the joint distribution of the predictand and its related model output random variable, namely the $p\left(y, y_{s}\right)$, as it was first shown by Krzysztofowic (1999) and many others later. Montanari and Brath (2004), under certain assumptions, carried out the predictive uncertainty assessment through the modeling of the joint distribution of the model errors and the model predictions $p\left(e, y_{s}\right)$ instead of modeling $p\left(y, y_{s}\right)$, although all of them formulated the problem by using a meta-Gaussian model (Kelly and Krzysztofowicz, 1997).

When we want to meet both objectives parameter estimation and predictive uncertainty assessment at the same time, we can address the issue through the modeling of the arising uncertainty from the uncertainty sources. For this task we can use two methods: the aggregate method or the disaggregate approach (Kavetski et al., 2006a, 2006b; Kuczera et al., 2006; Vrugt et al., 2008; Reichert and Mieleitner, 2009; Renard et al., 2010, 2011). The aggregate method, on which this research is focused, deals with modeling the hydrological model errors, considering the aggregated effects on them (i.e. bias, autocorrelation, heteroscedasticity, etc.), which are produced by all the uncertainty sources. This is made without the need to refer the particular contribution of each uncertainty source to these effects. If we are able to pack all the knowledge about the deviations of the model results from the observations, in an appropriate likelihood function (actually an error model), and the observed data meet the system observability conditions (Gelb, 1974), only then it will be possible a correct hydrological parameter estimation which yields unbiased, accurate and physically meaningful parameter values (Sorooshian and Gupta, 1983). This estimation necessarily needs to be a joint inference of error and hydrological models in order to avoid biased parameters (Evin et al., 2014). Moreover, since the modeling of the joint distribution $p\left(y, y_{s}\right)$ can be equivalent, under certain assumptions which will be exposed in the following section 2.2, to the modeling of the joint distribution of the errors and the model predictions $p\left(e, y_{s}\right)$, then a correct error model will also yield correct predictive uncertainty estimation. So, the answer to the question posed in the title of this section is that the closer we get to the ideal conditions for a correct error modeling, which may be a difficult task in hydrology (Smith et al., 2015), the more exact and accurate will be both the parameter estimation and the predictive uncertainty evaluation. 


\section{The error model}

The relation between a predictand $y$ and a model prediction $y_{s}$ can be defined as the composition of: i) a structural part which directly models the predictand expected dependence from the predictors, and ii) an additive or multiplicative random error $\varepsilon$. From the probability distribution of this random error, it is possible to derive the conditional probability distribution of the predictand. In fact, both distributions are the same when an additive error model is considered, namely $p\left(y-y_{s}, y_{s}\right)=p\left(e, y_{s}\right)$. When this is the case, we rely on the following relation:

$$
y=y_{s}\left(\left\{\boldsymbol{\theta}_{h}, \boldsymbol{\theta}_{e}\right\}, \tilde{\mathbf{X}}, \tilde{\mathbf{s}}_{0}\right)+e
$$

Equation (1) states that a predictand value $y$ is obtained through the sum of a model prediction $y_{s}$, and the error, "residual” or deviation $e$, which aggregates all sources of uncertainty. Model prediction, $y_{s}$, is expressed as a function of an observed set of “k” predictors (or model inputs) $\tilde{\mathbf{X}}=\left\{\tilde{\mathbf{x}}_{1: N}^{1}, \ldots, \tilde{\mathbf{x}}_{1: N}^{k}\right\}$, the set of hydrological and error model parameters $\left\{\boldsymbol{\theta}_{h}, \boldsymbol{\theta}{ }_{e}\right\}$, and the initial conditions $\tilde{\mathbf{s}}_{0}$.

The aggregated error $e$ can be decomposed in two components:

$$
e=\mu_{e \mid y_{s}}+\varepsilon
$$

The first one in Eq. (2) is a systematic component, $\mu_{e \mid y_{s}}$, generally modeled as deterministic, and which could be nonconstant. The second component $\varepsilon$, is a random variable with zero mean and whose variance could also be variable. It is very important to note that $\mu_{e \mid y_{s}}$ is an error shifting function, which is only able to represent the expectations of the error conditional distributions (the error conditional bias), namely $\mu_{e \mid y_{s}}=E\left[e \mid y_{s}\right]$, when the inferred errors fulfill the Total Expectation Law (TEL), also called the Iterated Expectations Law, or Adam's Law:

$$
E[e]=E\left\{E\left[e \mid y_{s}\right]\right\}
$$

where, $E[\bullet]$ is the expectation operator. In other words, Eq. (3) tells us that the marginal (total) expectation of the error is equal to the expectation of all error conditional (on $y_{s}$ ) expectations.

Besides, Eq. (1) could also be written as:

$$
y=E\left[y \mid y_{s}\right]+\varepsilon
$$

where $E\left[y \mid y_{s}\right]=y_{s}+\mu_{e \mid y_{s}}$ is the deterministic part of the predictand, and $\varepsilon$ is an additive random error. In the case that a hydrological model would yield unbiased outputs then $\mu_{e \mid y_{s}}=0$ and so $E\left[y \mid y_{s}\right]=y_{s}$. It is important to note that we can have a zero-mean error marginal distribution, but this is not equivalent to have hydrological model without bias, since conditional biases $\mu_{e \mid y_{s}} \neq 0$ could auto-compensate yielding a total expectation $E[e]=0$. Bias is a non-random deviation in the simulated $y_{s}$ value, due mainly to hydrological model structural deficiencies and systematic errors in forcing input data. 
In this research, and without loss of generality, the basin outlet discharge is considered as the simulated variable of predictive interest. In fact, this is the general practical situation and we have selected it as the only explicative variable for the bias. In particular, we have assumed a straightforward double linear bias model defined by:

$$
\begin{array}{lll}
\mu_{e \mid y_{s}}=\gamma & \text { if } & y_{s} \leq y_{0} \\
\mu_{e \mid y_{s}}=\gamma+\tau\left(y_{s}-y_{0}\right) & \text { if } & y_{s}>y_{0}
\end{array}
$$

where $\gamma, \tau$ and $y_{0}$ are error model parameters to be inferred jointly with the hydrological ones (in fact, all error model parameters will be jointly inferred with the hydrological ones). This double function aims at the consideration of two different expected error behaviors distinguishing the low flows (with a constant bias) from the high flows (with a linear bias).

As aforementioned, errors could exhibit a non-constant variance. Following Schoups and Vrugt (2010), Evin et al. (2013, 2014) and others, we assume in this paper a linear heteroscedasticity model, where $y_{s}$ is the only explicative variable for the conditional error standard deviation $\sigma_{e \mid y_{\mathrm{s}}}$. In this case, we can write:

$$
\sigma_{e \mid y_{s}}=\alpha+\kappa y_{s}
$$

where $\alpha$ and $\kappa$ are error model parameters. It is important to note that function $\sigma_{e \mid y_{\mathrm{s}}}$ represents to the standard deviation of the error conditional distributions, namely $\sigma_{e \mid y_{s}}^{2}=V\left[e \mid y_{s}\right]$, only if the inferred errors fulfill the Total Variance Law (TVL), also called the Variance Decomposition Law or Eve's Law:

$$
V[e]=E\left\{V\left[e \mid y_{s}\right]\right\}+V\left\{E\left[e \mid y_{s}\right]\right\}
$$

where $V[\bullet]$ is the variance operator. Equation (7) tells us that the marginal (total) variance of the error is equal to the sum of two terms: the first term assesses the expectation of the conditional (on $y_{s}$ ) variances of the error, and the second term evaluates the variance of the error conditional (on $y_{s}$ ) biases. On the contrary, when TVL is not fulfilled, function $\sigma_{e \mid y_{s}}$ does not represent to the standard deviation of the error conditional distributions, and an error standardization process by using this function would be a simple error scaling, instead of a correct standardization.

Having defined the models for the error bias and the error variance, we can also consider the possibility that the random component of the errors, $\varepsilon=e-\mu_{e \mid y_{s}}$, still exhibits serial correlation. Theoretically, the more accurate the bias model is, the smaller the remaining error serial correlation shall be. This dependence (error autocorrelation) can be due to the "memory" effect, caused by the propagation of forcing and structural errors through model storage components (Kavetski et al., 2003). The error autocorrelation can be modeled, as in Schoups and Vrugt (2010), Evin et al. (2013, 2014) and others, using an autoregressive (AR) model. At this stage the modification proposed by Evin et al. (2013) about the methodology applied in Schoups and Vrugt (2010) is considered: that is, errors should be studentized before applying an autoregressive error model on them. According to Evin et al. (2013), the reason for this change in the original method lies in the mathematical behavior of the autoregressive equations, and is particularly related to the error accumulation properties which are different in both approaches (for more details see Evin et al. (2013)). So, the standardized errors are defined as:

$$
\eta=\sigma_{e \mid y_{s}}^{-1}\left(e-\mu_{e \mid y_{s}}\right)=\sigma_{e \mid y_{s}}^{-1} \varepsilon
$$


where $\varepsilon$ is the zero-mean additive random error, $\sigma_{e \mid y_{s}}$ is the modeled standard deviation of the error conditioned on the $y_{s}$ value and $\mu_{e \mid y_{s}}$ is the modeled mean of the error (actually its bias) conditioned also on $y_{s}$.

Coming again to the matter of the errors autocorrelation, the equations of pure autoregressive model have the following compact form (Box and Jenkins, 1994):

$$
\left(1-\sum_{i=1}^{p} \phi_{i} B^{i}\right) \eta=\phi_{p}(B) \eta=z
$$

which expresses the $p$-order autoregressive model over the standardized errors $\eta$, where $\phi_{\mathrm{i}}$ are the $i$-order autoregressive coefficients, $B$ is the backshift operator $B^{i} \eta_{t}=\eta_{t-i}$ and $Z$ are the resulting innovations. Innovations are mutually independent random errors which represent the measurement errors (e.g. in forcing data and discharge measures). They follow a probability distribution with a constant variance $\sigma_{z}^{2}$. This variance, which should be considered as another (derived) parameter of the error model, can be evaluated according to the following expression (Box and Jenkins, 1994):

$$
\sigma_{z}^{2}=\sigma_{\eta}^{2}\left(1-\sum_{i=1}^{p} \rho_{i} \phi_{i}\right)
$$

where $\rho_{i}$ are the $i$-lagged autocorrelation coefficients of the standardized error series and $\sigma_{\eta}^{2}$ is the variance of the standardized errors. In this research we have used an AR(1) model as in Schoups and Vrugt (2010) and Evin et al. (2013, 2014). Since the innovations $Z$ are not standardized, they are subjected to a final transformation using their standard deviation:

$$
a=\sigma_{z}^{-1} z
$$

where $\sigma_{z}$ is obtained from Eq. (10) and $a$ are the final standardized innovations, which is an independent random variable with zero mean and unit variance (namely a standard white noise). Substituting Eq. (8) in Eq. (9) and this in Eq. (11) we obtain the relation between errors and innovations:

$$
a=\sigma_{z}^{-1} \phi_{p}(B)\left(\sigma_{e \mid y_{s}}^{-1}\left(e-\mu_{e \mid y_{s}}\right)\right)
$$

At this point, we should establish the pdf of the standardized innovations obtained in Eq. (12). This research models the standardized innovations using the Skew Exponential Power $\operatorname{SEP}(0,1, \xi, \beta)$ pdf, with two parameters (skewness $\xi$ and kurtosis $\beta$ ). The $\operatorname{SEP}(0,1, \xi, \beta)$ pdf, as shown in Schoups and Vrugt (2010), offers great flexibility by avoiding the a priori assumptions about specific forms of the innovations probability distribution. In fact, a $\operatorname{SEP}(0,1, \xi, \beta)$ pdf may adopt a variety of forms, from the Normal to Laplace distribution, as well as it is capable to reproduce asymmetries and heavy tails. The analytical expression of the $\operatorname{SEP}(0,1, \xi, \beta)$ pdf is defined as follows:

$$
p(a \mid \xi, \beta)=\frac{2 \sigma_{\xi}}{\xi+\xi^{-1}} w_{\beta} \exp \left(-c_{\beta}\left|a_{\xi}\right|^{\frac{2}{1+\beta}}\right)
$$

where 


$$
a_{\xi}=\xi^{-\operatorname{sign}\left(\mu_{\xi}+\sigma_{\xi} a\right)}\left(\mu_{\xi}+\sigma_{\xi} a\right)
$$

As summary, the vector of error model parameters is $\boldsymbol{\theta}_{e}=\left\{\alpha, \kappa, \gamma, \tau, y_{0}, \phi_{1}, \xi, \beta\right\}$ and $\sigma_{z}$ could be considered as a derived parameter. A major question related with the error model parameters must be pointed out. The application of the TLs on the error bias and variance models reduces the degrees of freedom in the Bayesian inference problem, and consequently, also reduces the number of independent error model parameters, namely the dimension of the parameter space. This will be clearly exposed in section 4.4 .

\subsection{Why and when is imperative the enforcement of the Total Laws}

Firstly, we want to emphasize a key concept which was previously exposed in the introduction: predictive uncertainty must be linked with the existence of a joint probability distribution of the predictand observations and the related model predictions. Under the hypothesis of an additive error model, this will be equivalent to considering the joint probability distribution of the predictand errors and the model predictions, as it was made for example in Montanari and Brath (2004), albeit they worked the statistics in the NQT (Kelly and Krzysztofowicz, 1997) transformed space. We are actually modeling the conditional distribution of the error, given the model prediction. In other words, we are modeling indirectly the above mentioned joint probability distribution of errors and model predictions. This means that the error conditional distributions must fulfill the proper restrictions, in order to ensure that all of them make up the full joint distribution. The enforcement of the Total Laws (TLs) exposed in Eqs. (3) and (7) allows us to impose the necessary restrictions in order to achieve that the conditional and marginal distributions of the inferred errors belong to the same bidimensional joint distribution of these errors and the modeled state variable of interest (the simulated streamflow in this research).

An incorrect error model will yield biased hydrological parameters (Sorooshian and Gupta, 1983) and, obviously, an incorrect uncertainty assessment. Two main causes can lead to this situation: i) an incorrect hypotheses about the error conditional distribution features (shape, mean and variance) or about the errors dependence structure; ii) making inferences without enforcing the TLs when we are modeling the error and state variable joint distribution through the definition of its conditional distributions. In order to better understand the TLs importance and implications, Fig. 1 shows what the TLs entail under different hypotheses for the error variance and bias models. 
Hydrol. Earth Syst. Sci. Discuss., doi:10.5194/hess-2017-9, 2017

Manuscript under review for journal Hydrol. Earth Syst. Sci.

Published: 17 January 2017

(c) Author(s) 2017. CC-BY 3.0 License.

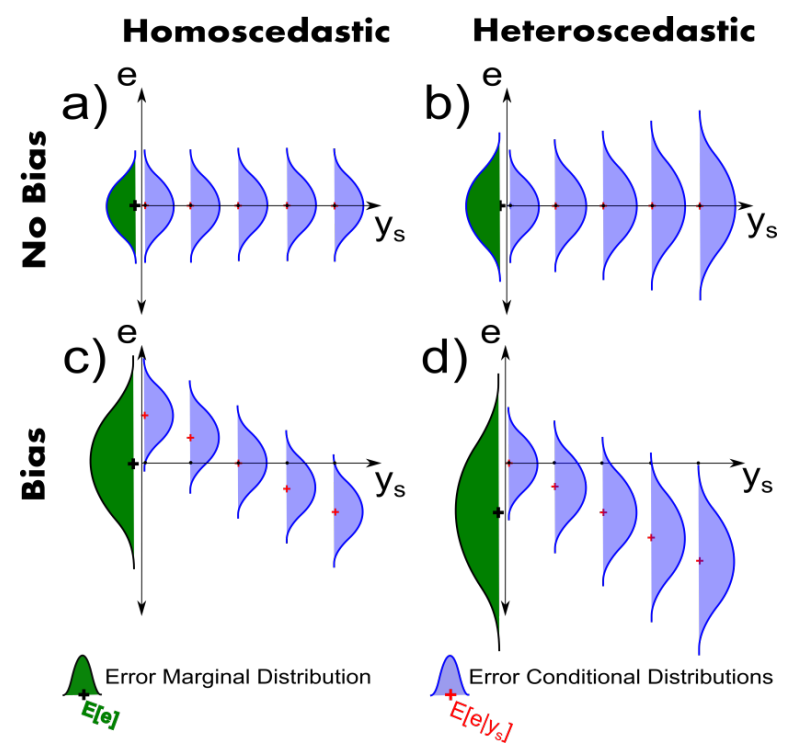

Figure 1. Schemes of decomposition of the state variable $\left(y_{s}\right)$ and its error joint distribution, in its conditional (blue) and marginal (green) distributions, under different bias and variance error models.

Fig. 1-(a) shows a scheme which is representative of the SLS error model, with the hypotheses of homoscedasticity (errors with constant variance) and also a constant zero-bias. Therefore, all conditional distributions are a "copy" of the error marginal distribution. Both hypotheses implicitly entail $E[e]=E\left[e \mid y_{s}\right]=0$ and $V[e]=V\left[e \mid y_{s}\right]$; i.e. the TLs given by Eqs. (3) and (7) are fulfilled, without the necessity of enforcing them.

Fig. 1-(b) represents the Weighted Least Squares error model (WLS), with non-constant error variance and constant zero-bias hypotheses. This scheme shows the conditional distributions assuming a linear relationship between the errors standard deviation and the simulated state variable (similar to that proposed in Eq. (6)). With WLS it is needed to explicitly enforce the TVL on the variance model, in order to get an error marginal distribution with a variance equal to the average variance of the error conditional distributions. For this particular error model (WLS), Eq. (7) collapses in the expression $V[e]=E\left\{V\left[e \mid y_{s}\right]\right\}$.

Fig. 1-(c) shows the case of having errors with a constant conditional variance and a non-constant linear bias. In this case, the marginal distribution is different from the conditional ones, although all these have the same shape. This is due to the nonconstant bias which affects the position of the conditional distributions. Moreover, it can occur (as in the example in Fig. 1(c)) the effect of errors autocompensation: the marginal distribution shows a zero-mean value whereas the conditional distributions exhibit bias. In this case, a non-constant conditional bias means that Eq. (3) for the TEL is not automatically fulfilled (must be enforced). With a constant conditional variance, the TVL in Eq. (7) simplifies to $V[e]=V\left[e \mid y_{s}\right]+V\left\{E\left[e \mid y_{s}\right]\right\}$.

Finally, Fig. 1-(d) shows the general case with both non-constant error conditional variances and bias. In this case, we must enforce on both variance and bias models the general expressions of the TLs (Eqs. (3) and (7)). Section 4 describes the inference settings for the case study and how the TLs are applied on each of them. On the contrary, section 6.1 will show the consequences of not enforcing the TLs. 


\subsection{Parameter uncertainty}

In Bayesian inference, it is necessary to construct a probability model based on the observed data of the variable of interest, as well as on the model parameters. Formal inference requires the setting up of a joint probability model to take all these random variables (i.e. observed state variables and model parameters) into account. Therefore, given the hydrological and error models, $\mathfrak{M}_{h, e}$, and also given the set of initial conditions, $\tilde{\mathbf{s}}_{0}$, and an observation of the "k" predictors, $\tilde{\mathbf{X}}=\left\{\tilde{\mathbf{x}}_{1: N}^{1}, \ldots, \tilde{\mathbf{x}}_{1: N}^{k}\right\}$, it must be constructed $p\left(\tilde{y},\left\{\boldsymbol{\theta}_{h}, \boldsymbol{\theta}_{e}\right\}\right)$, the joint distribution of observations of the state variable of interest, $\tilde{y}$, and the hydrological and error model parameters, $\left\{\boldsymbol{\theta}_{h}, \boldsymbol{\theta}_{e}\right\}$. This joint distribution, also called "the full Bayesian model” (Bolstad, 2010), is conditioned on $\mathfrak{M}_{h, e}, \tilde{\mathbf{s}}_{0}, \tilde{\mathbf{X}}$ and is formed by taking the product of two probability distributions:

$$
p\left(\tilde{y},\left\{\boldsymbol{\theta}_{h}, \boldsymbol{\theta}_{e}\right\}\right)=p\left(\tilde{y} \mid\left\{\boldsymbol{\theta}_{h}, \boldsymbol{\theta}_{e}\right\}\right) p\left(\left\{\boldsymbol{\theta}_{h}, \boldsymbol{\theta}_{e}\right\}\right)=p\left(\left\{\boldsymbol{\theta}_{h}, \boldsymbol{\theta}_{e}\right\} \mid \tilde{y}\right) p(\tilde{y})
$$

Then, based on Eq. (1) that hypothesizes an additive error model, we have $p\left(\left\{\boldsymbol{\theta}_{h}, \boldsymbol{\theta}_{e}\right\} \mid \tilde{y}\right)=p\left(\left\{\boldsymbol{\theta}_{h}, \boldsymbol{\theta}_{e}\right\} \mid \varepsilon\right)$, where $\varepsilon$ is the random error solved from Eq. (2). The analytical expression for $p\left(\varepsilon \mid\left\{\boldsymbol{\theta}_{h}, \boldsymbol{\theta}_{e}\right\}\right)$ is called the sampling distribution, when the parameters are considered as known. The same analytical expression is called the parameter likelihood function $\ell\left(\left\{\boldsymbol{\theta}_{h}, \boldsymbol{\theta}_{e}\right\} \mid \varepsilon\right)$, when the known variables are the observed data. The likelihood function incorporates the effect of the data on the prediction model, namely on the hydrological and error models jointly considered. Besides, the parameter prior distribution $p\left(\left\{\boldsymbol{\theta}_{h}, \boldsymbol{\theta}_{e}\right\}\right)$ describes the a priori knowledge we have about the hydrological and error models. Then, by applying Bayes' Rule in its unscaled form on Eq. (15), the parameter posterior distribution is given by:

$$
p\left(\left\{\boldsymbol{\theta}_{h}, \boldsymbol{\theta}_{e}\right\} \mid \varepsilon\right) \propto \ell\left(\left\{\boldsymbol{\theta}_{h}, \boldsymbol{\theta}_{e}\right\} \mid \varepsilon\right) p\left(\left\{\boldsymbol{\theta}_{h}, \boldsymbol{\theta}_{e}\right\}\right)
$$

Since in section 2.2 we have already modeled the errors, we can derive from it the analytical expression for the likelihood function. This task is detailed in Appendix A, where the following log-likelihood function is obtained:

$$
\mathcal{L}\left(\left\{\boldsymbol{\theta}_{h}, \boldsymbol{\theta}_{e}\right\} \mid \varepsilon\right) \cong N \log \frac{2 \sigma_{\xi} w_{\beta}}{\sigma_{\mathbf{z}}\left(\xi+\xi^{-1}\right)}-\sum_{t=1}^{N} \log \sigma_{\varepsilon \mid y_{s}}-c_{\beta} \sum_{t=1}^{N}\left|a_{\xi}\right|^{\frac{2}{1+\beta}}
$$

where: $\left\{\boldsymbol{\theta}_{h}, \boldsymbol{\theta}_{e}\right\}$ is the set of hydrological and error model parameters; $\varepsilon$ is the random error; $\sigma_{\varepsilon \mid q_{s}}$ is the conditional standard deviation of these random errors, which can be estimated by Eq. (6); $\sigma_{z}$ is the standard deviation of innovations, estimated with Eq. (10); the terms $\mu_{\xi}, \sigma_{\xi}, c_{\beta}, w_{\beta}$ are a function of the skewness $\xi$ and kurtosis $\beta$, parameters of the SEP distribution (more details in Schoups and Vrugt (2010)); $\boldsymbol{a}_{\xi}$ is a function of the standardized innovations given by Eq. (14); and $N$ is the number of observations.

Parameter posterior pdf describes the probability density that the model parameters assume particular values conditioned on the observed data. I.e. it describes the parameter uncertainty. In this study, both hydrological and error model parameters are conditioned simultaneously (joint inference) on observations. Computational sampling of the posterior is made through a Markov chain Monte Carlo (MCMC) algorithm. Modern MCMC algorithms, which are an evolution of the original 
Metropolis algorithm (Metropolis and Rosenbluth, 1953), are currently used to "calibrate” the model parameters and to assess the uncertainties of these parameter estimates. Even high-dimensional and multimodal posterior distributions can be efficiently sampled by these recent MCMC algorithms after convergence of the chain (or chains) has been reached (Cowles and Carlin, 1996). The Differential Evolution Adaptive Metropolis algorithm DREAM-ZS (Schoups and Vrugt, 2010; Laloy and Vrugt, 2012) has been selected in this research to sample the posterior distribution of the parameters. The DREAM-ZS algorithm is a modification of the DREAM (Vrugt et al., 2008, 2009a) algorithm. DREAM-ZS simultaneously runs multiple Markov chains to explore the whole parameter space, uses an archive of past states to generate candidate points in each individual chain and automatically adjusts the scale and orientation of the proposal distribution. Such sampling is more efficient than an optimal random walk Metropolis. The R-statistic has been used to check whether the chains have converged, with a threshold value of $\mathrm{R}$ equal to 1.2 (Gelman and Rubin, 1992). In this research, the number of chains used in the inference configurations was 10 .

\subsection{Predictive Uncertainty}

One of the main advantages to pursuing a formal method of Bayesian inference is the ability to make a correct estimate of the predictive uncertainty. Given the hydrological and error models, $\mathfrak{M}_{h, e}$, a formal definition of the predictive uncertainty for the prediction in a period with $N$ time steps, adapted from Mantovan and Todini (2006) is given by the next equation:

$$
p\left(y \mid y_{s}\left(\tilde{\mathbf{y}}, \tilde{\mathbf{X}}, \tilde{\mathbf{s}_{0}}\right)\right)=\int_{\Theta} p\left(y \mid y_{s}\left(\left\{\boldsymbol{\theta}_{h}, \boldsymbol{\theta}_{e}\right\}, \tilde{\mathbf{X}}\right)\right) p\left(\left\{\boldsymbol{\theta}_{h}, \boldsymbol{\theta}_{e}\right\} \mid \tilde{\mathbf{y}}, \tilde{\mathbf{X}}, \tilde{\mathbf{s}}_{0}\right) d \boldsymbol{\theta}
$$

where the left term $p\left(y \mid y_{s}\left(\tilde{\mathbf{y}}, \tilde{\mathbf{X}}, \tilde{\mathbf{s}}_{0}\right)\right)$ is the probability density of the predictand $y$, conditioned upon its $N$ observations $\tilde{\mathbf{y}}$, the set of initial conditions $\tilde{\mathbf{s}}_{0}$, the observed predictors $\tilde{\mathbf{X}}$, and necessarily also on $\mathfrak{M}_{h, e}$. Hence, the dependence on the parameters has been marginalized integrating over the entire domain of existence of these parameters; i.e., the ensemble of all possible parameter realizations. In other words, for assessing the predictive distribution (hereinafter PD) of the predictand, one has to take into account all possible model predictions (namely one per parameter vector realization), instead of only considering the prediction which corresponds to the most plausible parameter set. The first right term $p\left(y \mid y_{s}\left(\left\{\boldsymbol{\theta}_{h}, \boldsymbol{\theta}_{e}\right\}, \tilde{\mathbf{X}}\right)\right)$ is the probability density of the predictand conditioned on the observed predictors and a unique set of parameters. The last right term $p\left(\left\{\boldsymbol{\theta}_{h}, \boldsymbol{\theta}_{e}\right\} \mid \tilde{\mathbf{y}}, \tilde{\mathbf{X}}, \tilde{\mathbf{s}}_{0}\right)$ is the posterior of the parameters given the observed predictors, the observed predictand and the initial conditions. This probability distribution can be derived by means of a Bayesian inferential process (as explained in section 3.1), and it is used to marginalize out the conditionality on the parameters of the prediction.

The way of computing the PD is as follows. First a unique set $\left\{\boldsymbol{\theta}_{h}, \boldsymbol{\theta}_{e}\right\}$, of hydrological and error model parameters, is sampled from their posterior distribution. After the parameter posterior sampling, the hydrological model is run for the sampled hydrological parameters $\boldsymbol{\theta}_{h}$, getting a model simulation of the predictand $y_{s}\left(\boldsymbol{\theta}_{h}, \tilde{\mathbf{X}}\right)$. Then, by using repeatedly the prediction sampling equation given by:

$$
\hat{y}=y_{s}\left(\boldsymbol{\theta}_{h}, \tilde{\mathbf{X}}\right)+\mu_{e \mid y_{s}}+\sigma_{e \mid y_{s}} \sigma_{z} \phi_{p}^{-1}(B) a
$$

we get the probability density of the predictand, conditioned upon a unique set of hydrological and error model parameters, namely $p\left(y \mid y_{s}\left(\left\{\boldsymbol{\theta}_{h}, \boldsymbol{\theta}_{e}\right\}, \tilde{\mathbf{X}}\right)\right)$, where $\hat{y}$ is an estimated prediction of the predictand, conditioned on the previously 
obtained model simulation $y_{s}\left(\boldsymbol{\theta}_{h}, \tilde{\mathbf{X}}\right)$ (i.e. on the hydrological model) and also conditioned on a unique realization from the error model. This error model realization is obtained through the previously sampled error model parameters $\boldsymbol{\theta}_{e}$, using a random extraction $\boldsymbol{a}$ from the SEP innovations distribution. To define with enough resolution this pdf of the predictand conditioned on $y_{s}\left(\boldsymbol{\theta}_{h}, \tilde{\mathbf{X}}\right)$, a sufficient number "s" of prediction samples $\hat{\mathbf{Y}}=\left\{\hat{\mathbf{y}}_{1: N}^{1}, \ldots, \hat{\mathbf{y}}_{1: N}^{s}\right\}$ must be obtained with the corresponding "s" random extractions from the SEP innovations distribution. Finally, all previous steps are repeated over multiple hydrological and error parameter posterior samples $\left\{\boldsymbol{\theta}_{h}, \boldsymbol{\theta}_{e}\right\}_{i=1, \ldots, m}$, generating an ensemble of predictions $\{\hat{\mathbf{Y}}\}_{i=1, \ldots, m}$, in which the dependence on the parameters has been marginalized. The present paper considers the mean of that ensemble of predictions as the estimator for the expected prediction $\hat{\mathbf{y}}^{*}$, unconditioned to the parameters, formally:

$$
E\left[y \mid y_{s}\left(\tilde{\mathbf{y}}, \tilde{\mathbf{X}}, \tilde{\mathbf{s}}_{0}\right)\right] \simeq \hat{\mathbf{y}}^{*}=\frac{1}{m s} \sum_{i=1}^{m} \sum_{j=1}^{s} \hat{\mathbf{y}}_{1: N}^{i, j}
$$

\section{Application to Rainfall-Runoff modeling}

This paper considers the simulated daily streamflow $q_{s}$, as the state variable of interest to be predicted. The key concept is that, this state variable and its error belong to a bivariate population with a unique joint pdf. This belonging relationship imposes restrictions which must be fulfilled by both the state variable and the errors; actually by the parameters of hydrological and error models. That is to say, the error and hydrological inferred parameters, which generally are only conditioned by the observed data through the likelihood function, will also be conditioned by this belonging relationship expressed by the TLs in Eqs. (3) and (7). So, this section shows how to correctly perform the parameter joint inference of hydrological and error models, in order to reach the fulfillment of the TLs.

\subsection{The basin}

We have selected the French Broad River (hereinafter FB) basin. It is located in North Carolina (USA) and has an area of $2448 \mathrm{~km}^{2}$. FB basin information can be obtained from the Model Parameter Estimation Experiment (MOPEX) data set (Duan et al., 2006). Besides, FB basin is a broadly known basin by the hydrologists' community. Previous research related with this paper also performed inferences in this basin, as the works of Schoups and Vrugt (2010) or Evin et al. (2013, 2014). This basin is a representative humid catchment where actual evapotranspiration is energy-limited, hence runoff production opportunity is high: FB basin has a mean annual rainfall of $1495 \mathrm{~mm}$, and a mean annual potential evapotranspiration of 820 mm. Five years (from 01/01/1962 to 31/12/1966) of observed daily forcings (precipitation and potential evapotranspiration) and observed daily streamflow were used to identify hydrologic and error model parameters. The two previous years were used as warm-up period.

\subsection{The hydrological models}

Two hydrological models have been chosen to illustrate the theoretical issues on which this research deals with. The reason for the election is mainly their use in researches related with the present paper.

The first one is the CRR model, which was also used in Schoups and Vrugt (2010) or Schoups et al. (2010). CRR is a conceptual spatially lumped hydrologic model for the simulation of daily rainfall-runoff processes, and it has seven parameters to calibrate: the maximum interception (Imax, units of $\mathrm{mm}$ ), the Soil water storage capacity $\left(\mathrm{S}_{\max }\right.$, units of $\mathrm{mm}$ ), the maximum percolation rate $\left(\mathrm{Q}_{\mathrm{smax}}\right.$, units of $\left.\mathrm{mm} / \mathrm{d}\right)$, the evaporation parameter ( $\alpha_{\mathrm{e}}$, dimensionless), the runoff parameter ( $\alpha_{\mathrm{f}}$, dimensionless), the fast reservoir constant $\left(\mathrm{K}_{\mathrm{f}}\right.$, units of days) and the slow reservoir constant ( $\mathrm{K}_{\mathrm{s}}$, units of days). The model considers the processes of interception, throughfall, evapotranspiration, runoff generation, percolation, and surface and subsurface routing of water to the basin outlet. 
The second model is the well-known GR4J. It is also spatially lumped and was developed to provide, on average, good performance across a wide range of catchment conditions (Perrin et al., 2003). The GR4J model, with only four parameters, is a more parsimonious model than CRR. These four parameters are: the production store capacity $\left(\theta_{1}\right.$, units of mm), the groundwater exchange coefficient $\left(\theta_{2}\right.$, units of $\left.\mathrm{mm}\right)$, the one day-ahead maximum capacity of the routing store $\left(\theta_{3}\right.$, units of $\mathrm{mm})$ and the base time of the unit hydrograph $\left(\theta_{4}\right.$, units of days).

In order to infer the parameter posteriors (as explained in section 3.1), we have assumed non-informative Priors. The assumption of uniform Priors for all parameters is acceptable since a sufficient number of daily data points $(N=1825)$ supports the parameter inference.

An important question to be remarked is that snow accumulation and snowmelt are not accounted for in any of both models, although these processes occur in the FB basin, but with low significance. Therefore, it is expected that this model misspecification will have a non-severe influence in the results, but also an undermining of either the reliability of the inferred parameters or the reliability of the prediction. These consequences will be evaluated in the performed inferences.

\subsection{Implemented error models}

In this paper, we are going to perform different inferences considering four error models which, sorted in crescent order of complexity, are: SLS, WLS, GL++ and GL++Bias. Besides, for the WLS and GL++ error models, the inferences will be also performed with and without the enforcement of the TLs. This later inferences are called NTL inferences and they are an illustrative example of the problems which can arise from the joint inference when TLs are neglected.

The SLS (Standard Least Squares) error model is the standard approach in regression analysis. For SLS, the errors are a zeromean measurement random noise, so they are serially independent and, normally and identically distributed. SLS is considered as the reference inference, since it is the most common method in parameter estimation.

The WLS approach (Weighted Least Squares) considers all the same hypotheses that SLS, except that WLS applies a heteroscedastic error model which weights the errors by their standard deviation as in Eq. (6). WLS needs to apply the TVL. However, section 6.1 will show what happens with the WLS inference without the enforcement of the TVL (which is called WLS-NTL inference).

The GL++ is an evolution of the GL error model described in Schoups and Vrugt (2010). The modifications on GL are two. Firstly we have included the recommendation given by Evin et al. (2013): errors must be studentized before applying an autoregressive model on them. Secondly, the joint inference has taken into account the enforcement of the TLs. Therefore, GL++ assumes zero-mean errors and the same heteroscedasticity model as in the WLS, but relaxes the hypothesis of error independence through a first order AR model (Eq. (9)). The resulting innovation has a variance given by Eq. (10) and is distributed according to a SEP distribution described by Eqs. (11) to (14). The Log-likelihood function in Eq. (17) includes all these assumptions, assuming an unbiased conditional error. Again, section 6.1 will show the results of the GL++NTL inference, in order to illustrate what occurs to this kind of error model when TVL is not enforced.

Finally, the GL++Bias error model assumes all hypotheses of GL++, but relaxes the hypothesis of having zero-mean conditional error distributions. A bias model is hypothesized according to Eq. (5), which tries to correct these conditional biases. Therefore, GL++Bias is the full error model described in section 2.2.

\subsection{Enforcement of the Total Laws}

On all the above described error models, it will be applied the enforcement of either one or both TLs, except on SLS (because it is not needed) and on the NTL cases. In the case of WLS and GL++ approaches, they must yield inferred errors which fulfill the simplified expression of the TVL given by:

$$
V[e]=E\left\{V\left[e \mid q_{s}\right]\right\}
$$


This conditioning to TVL allows us to reduce one degree of freedom in the inference problem: the intercept $\alpha$ from the heteroscedasticity linear model will be a function of other parameters and the state variable. So, this error parameter does not need to be sampled in the computational Bayesian inference process. Following the calculations in Appendix B, we obtain:

$$
\alpha=\left(V[e]-\kappa^{2} V\left[q_{s}\right]\right)^{0.5}-\kappa E\left[q_{s}\right]
$$

Eq. (22) is the condition that must meet the intercept parameter $\alpha$ in order to achieve that the inferred errors with the WLS and GL++ approaches, are able to fulfill the TVL.

In the case of the GL++Bias error model, both error variances and bias are supposed to be non-constant. In this case we must enforce on both variance and bias models the general expressions of the TLs given by Eqs. (3) and (7). In this way, this enforcement (as shown in Appendix B) allows us to set the following four parameters:

For the linear heteroscedasticity model given by Eq. (6),

i) The intercept parameter, $\alpha$

$$
\alpha=\left(V\left[e_{1}\right]-\kappa^{2} V\left[q_{s_{1}}\right]\right)^{0.5}-\kappa E\left[q_{s_{1}}\right]
$$

ii) The slope parameter, $\kappa$

$$
\kappa=\left(\frac{V\left[e_{1}\right]-V\left[e_{2}\right]+\tau^{2} V\left[q_{s_{2}}\right]}{E\left[q_{s_{1}}^{2}\right]-E\left[q_{s_{2}}^{2}\right]}\right)^{0.5} \quad \text { for } \quad \alpha \approx 0
$$

It is important to note that the later equation, which yields $\kappa$ explicitly, is only valid for small values of parameter $\alpha$. If not, parameter $\kappa$ should be calculated in an iterative way by solving Eq. (B13).

And for the double linear bias model given by Eq. (5),

iii) The intercept parameter, $\gamma$, for the low-flows population

$$
\gamma=E\left[e_{1}\right]
$$

iv) The slope parameter, $\tau$, for the high-flows population

$$
\tau=\frac{E\left[e_{1}\right]-E\left[e_{2}\right]}{q_{0}-E\left[q_{s_{2}}\right]}
$$

where the subindexes 1 and 2 distinguish the low and high streamflow populations respectively. The four Eqs. (23) to (26) allow that inferred errors by GL++Bias be able of fulfilling both TLs. Additionally, these imposed equations remove four degrees of freedom from the inference problem. That is to say, GL++Bias has only four (and not eight) free error model parameters to be sampled by the MCMC algorithm. These free parameters are: the bias parameter $q_{0}$, the SEP parameters $\beta$ and $\xi$, and the autoregressive coefficient $\phi_{1}$. 
Hydrol. Earth Syst. Sci. Discuss., doi:10.5194/hess-2017-9, 2017

\section{Results}

\subsection{The reference inference: SLS}

The target of the SLS method is the mean squared error minimization of the prediction, and errors are assumed to be independent, homoscedastic and Gaussian with zero-mean. Therefore, a SLS calibrated hydrological model must yield the best fitting to the observed values. With this aim, the SLS method tries to compensate the model misspecifications and the data errors through the parameter values, by a departure from their "true" value. We consider the parameter "true" values as the generally unknown parameter set, which would be inferred in a calibration without model structural deficiencies or biased data errors: i.e. only with random measurement errors.

SLS yields error marginal variances in the case study for both hydrological models $\left(0.34 \mathrm{~mm}^{2} / \mathrm{d}^{2}\right.$ and $0.43 \mathrm{~mm}^{2} / \mathrm{d}^{2}$ for CRR and GR4J respectively) which are the minimum reached in all performed inferences (compare RMSE values in Table 1). This situation can be expected, since this is the way to reach the target of the SLS inference. Another interesting feature is the (marginal) mean of the errors, which is not zero (i.e., SLS unbiased errors hypothesis is not fulfilled), and it is slightly greater with CRR than with GR4J (-0.11 mm/d and $-0.02 \mathrm{~mm} / \mathrm{d}$ respectively). In fact, as it was also expected, SLS inference does not fulfill any of its underlying hypotheses about the errors (Fig. 2): normality, homoscedasticity and independence. Regarding the normality assumption (Fig. 2, top panels), the error distribution has an excess of kurtosis, for both hydrological models. The non-compliance of the homoscedasticity is clearly shown in middle panels of Fig. 2: the funnel-shaped scatter plot of the standardized errors versus the simulated streamflow shows that error variance varies with the streamflow magnitude. To assess this heteroscedastic property numerically, it has been calculated a moving variance of the standardized errors, by taking a moving window through the scatter plot, with a length of 60 data, over which the variance is calculated. The result of this calculation is the red line drawn over the scatter. The flatness of this line would indicate the homoscedasticity of the errors and the line is far to be flat. Finally, the errors independence hypothesis can be judged through the autocorrelation function shown in Fig. 2 (bottom panels). These graphs also show the 95\% Anderson's limits (Anderson, 1942) for null correlation hypothesis. As it can be observed, SLS yields a clear not null autocorrelation for the first ten lags, for both hydrological models.

Table 1 shows the Log-likelihood value for each of the inferred error models and it is clear that the worst values are for SLS (-1625 with CRR and -1819 with GR4J hydrological model). On the contrary, as shown in Tables 2 and 3, SLS generally (if the parameters are identifiable) yields the parameter estimates with the lowest uncertainty: namely, with the smallest sample coefficients of variation (CV). In this case study, GR4J model shows perfectly identifiable inferred parameters and with little uncertainty. However, CRR model shows two non-identifiable parameters, $\alpha_{e}$ and $\alpha_{f}$, which are highly uncertain parameters in comparison with the other in SLS (see Table 2), showing CVs of 0.20 and 1.77 respectively.
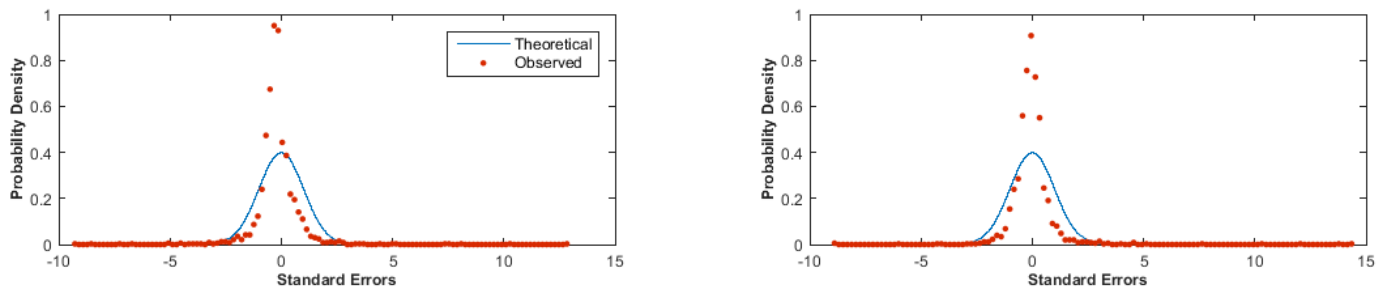
Hydrol. Earth Syst. Sci. Discuss., doi:10.5194/hess-2017-9, 2017

Manuscript under review for journal Hydrol. Earth Syst. Sci.

Published: 17 January 2017

(c) Author(s) 2017. CC-BY 3.0 License.
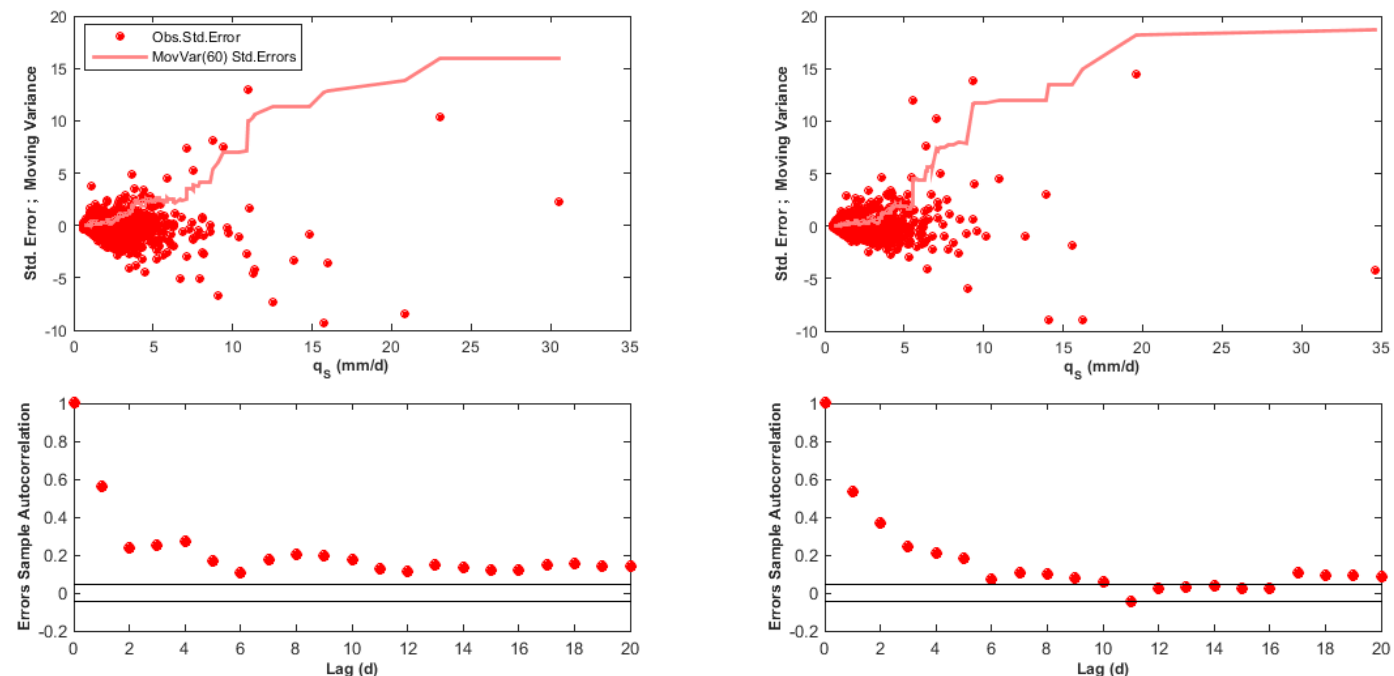

Figure. 2. Test of SLS error model hypotheses for CRR (left column) and GR4J (right column) hydrological models: normality of observed standard errors in top panels, homoscedasticity evaluation of errors in middle panels and independence assessment of the errors through the autocorrelation function in bottom panels (the 95\% Anderson's limits in solid black lines).

Concerning the performance of the mean prediction, Table 1 shows that SLS has the highest NSE index among all inferred error models. On the one hand, it results a NSE $=0.90$ in CRR, whereas GR4J yields a NSE $=0.87$. On the other hand, CRR shows a Volume Error, defined as $V E=\left(V_{s}-V\right) V^{-1}$, larger than the obtained with GR4J model. This result is coherent with the value for the marginal mean of the errors obtained for each model. In Fig. 3 are represented the observations $\tilde{q}$, the mean prediction $\hat{q}^{*}$ and the hydrological model prediction for the Maximum a Posteriori (MAP) parameter set $q_{s_{\text {MAP }}}$. This figure shows how CRR yields a nice fitting to the largest peakflow of the calibration period, but CRR obtains a moderate fitting to the rest of peakflows and a general overestimation of the low flows. A similar general behavior is achieved by GR4J model, as can be seen in the same Fig. 3.

The validation of the predictive uncertainty for both hydrological models can be visually checked in Fig. 3: this figure shows the $95 \%$ prediction uncertainty band, resulting with a constant width for all the streamflow magnitudes (although a merely visual effect seems to contradict this). It is important to note that Fig. 3 also shows the parameter uncertainty band, but its extreme thinness means that, in this inference, the contribution of the parameter uncertainty to the total uncertainty can be neglected. the predictand with the PP-Plots tool (Laio and Tamea, 2007; Thyer et al., 2009; Renard et al., 2010). In Fig. 4 (left panel) the blue line shows a systematic bias (overprediction) with an uncertainty overestimation for the CRR model. In the same Fig. 4 (right panel), a very small overpredictive bias and also an uncertainty overestimation is shown for the GR4J model when SLS inference is used. 
Hydrol. Earth Syst. Sci. Discuss., doi:10.5194/hess-2017-9, 2017

Manuscript under review for journal Hydrol. Earth Syst. Sci.

Published: 17 January 2017

(c) Author(s) 2017. CC-BY 3.0 License.
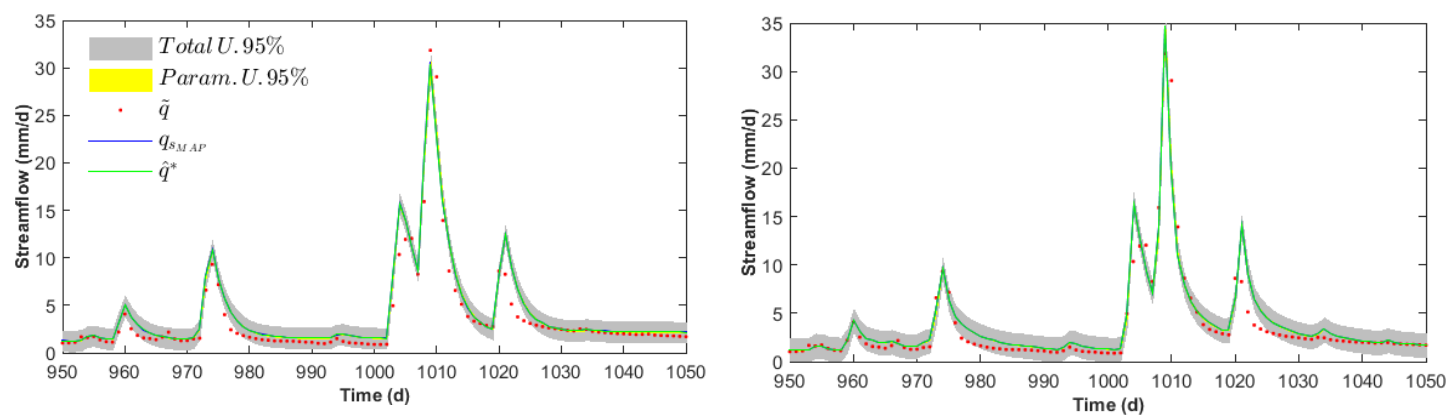

Figure 3. Predictive performance and $95 \%$ uncertainty bands (parameter and total) with SLS inference on CRR (left) and GR4J
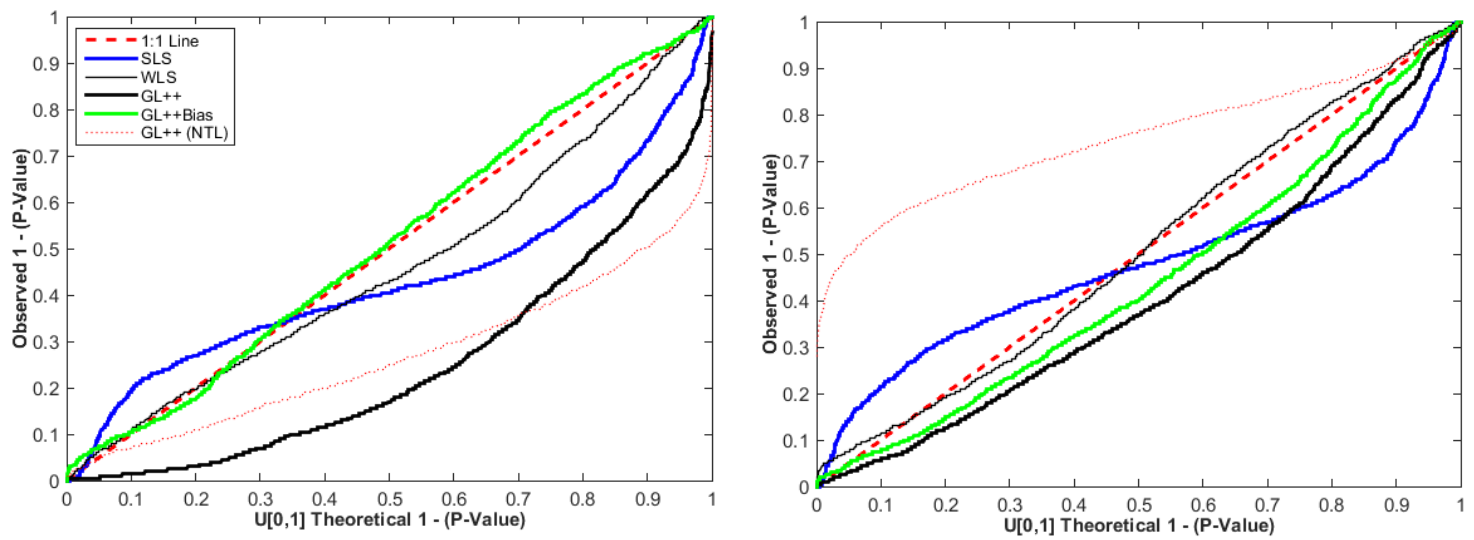

Figure 4. PP-Plots of the PDs for all performed inferences with CRR (left) and GR4J (right) models.

494

495

496

497

498

499

500

501

502

503

504

\subsection{WLS inference}

In this case study, the WLS inference does not fulfill any of its error model hypotheses, as SLS, with the exception of the homoscedasticity hypothesis. As in SLS inference, the errors are not Normal (Fig. not shown). The good variance stabilization of the inferred errors with both hydrological models can be observed in top panels of Fig. 5, where are depicted the scatter plots for the standard errors and for the stabilized errors. Over the scatters there are also two lines representing the corresponding moving variances, calculated as it was explained for the SLS inference. As it can be observed, the calculated moving variance for the errors has the same appearance as in SLS. But, the moving variance for the stabilized errors is practically a horizontal line; i.e., it represents a constant variance. However, bottom panels in Fig. 5 show how the stabilized errors have a higher autocorrelation than the raw errors. In other words, WLS solves the errors heteroscedasticity problem, but increases the problem of error serial dependence. The fulfillment of the homoscedasticity hypothesis is able to improve the Log-likelihood respect to SLS, in a 72\% for CRR model whereas this improvement with GR4J is about 60\% (Table 1). 

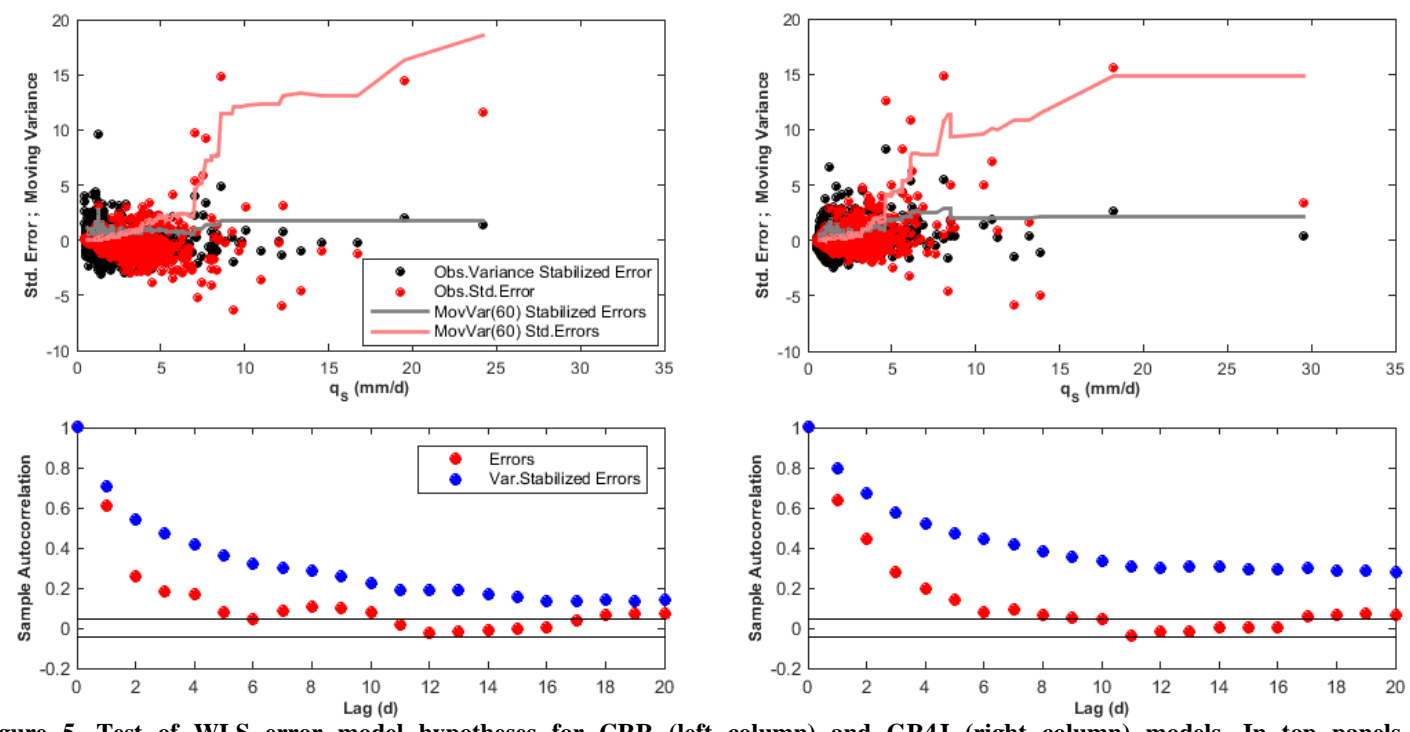

Figure 5. Test of WLS error model hypotheses for CRR (left column) and GR4J (right column) models. In top panels, homoscedasticity evaluation for the observed errors (red dots and red line) and for the variance stabilized errors (black dots and grey line). In bottom panels, the independence assessment for observed errors and for the variance stabilized errors, through the autocorrelation function (the $\mathbf{9 5 \%}$ Anderson's limits in solid black lines).

509

The performance of the WLS mean prediction and the negligible contribution of the parameter uncertainty to the total uncertainty are very similar to the SLS one (graphs not shown). Comparing both inferences in Table 1, WLS exhibits a slightly worse NSE index, but a better VE. The reduction in NSE is due to the fact that WLS gives a minor weight to the high flows during the calibration, yielding a poorer fit to the peakflows.

An improvement with respect to SLS is that WLS has a very good prediction uncertainty assessment, with GR4J slightly better than with CRR, as it is shown in the PP-Plots of Fig. 4. But, the fact that WLS does not fulfill the error independence hypothesis implies that: i) there is a problem with the hydrological model structure and/or with the data reliability; and ii) we have a good uncertainty assessment of a distorted hydrological model which is being used with biased inferred parameters. It is expected that, out of the calibration period, these biased parameters will induce a poor performance of the hydrological model as well as of the uncertainty assessment. This was called model divergence phenomenon by Sage and Melsa (1971) and Sorooshian and Dracup (1980), long time ago.

\subsection{GL++ inference}

The inference with the GL++ error model fulfills practically all its hypotheses with both CRR and GR4J hydrological models. As it is shown in top panel of Fig. 6, CRR presents an error distribution with heavier tails than GR4J, but despite this difference, the fitting to the SEP distribution is good and similar for both hydrological models. The error autocorrelation is almost eliminated with both hydrological models, although as shown in Fig. 6 (bottom panel), still remains a very small but significant negative autocorrelation of the innovations for the first three lag times.

Regarding the variance stabilization, it can be seen in Fig. 6 (middle panel) how the conditional variance of errors and innovations (represented as previously explained by a moving sample variance) are very similar almost for all the range of simulated streamflows. Hence, GL++ is not able to stabilize the error variances as WLS does it. The reason of this poor behavior of the variance stabilization is the error bias which has arisen with this GL++ inference, which is not modeled. This overpredictive bias can be clearly noticed for the CRR model: standard errors (red dots in the middle panel of Fig. 6) have an increasing, with the simulated streamflow magnitude, negative mean. This effect can also be observed in PP-Plots of Fig. 4, 
where for the GL++ inference the curvature is pronounced and far away from an "S-shape". Therefore, the GL++ error model infers the bivariate distribution $p\left(e, q_{s}\right)$ considering the position of all its error conditional distributions centered at zero (i.e. with a null conditional bias) and given this, it estimates its shape properties (e.g. its variance). The result is a poor performance of the variance model given by Eq. (6), which is not able to fit the correct amplitude of the bivariate distribution without considering correctly its location. In short, in presence of a non-constant bias, a variance model on its own is not able to correctly model the bivariate distribution of the simulated discharges and its errors. The expression of the TVL in Eq. (7) summarizes this idea, since it considers both conditional bias and conditional variance terms to obtain the total (marginal) variance of the hydrological model errors.
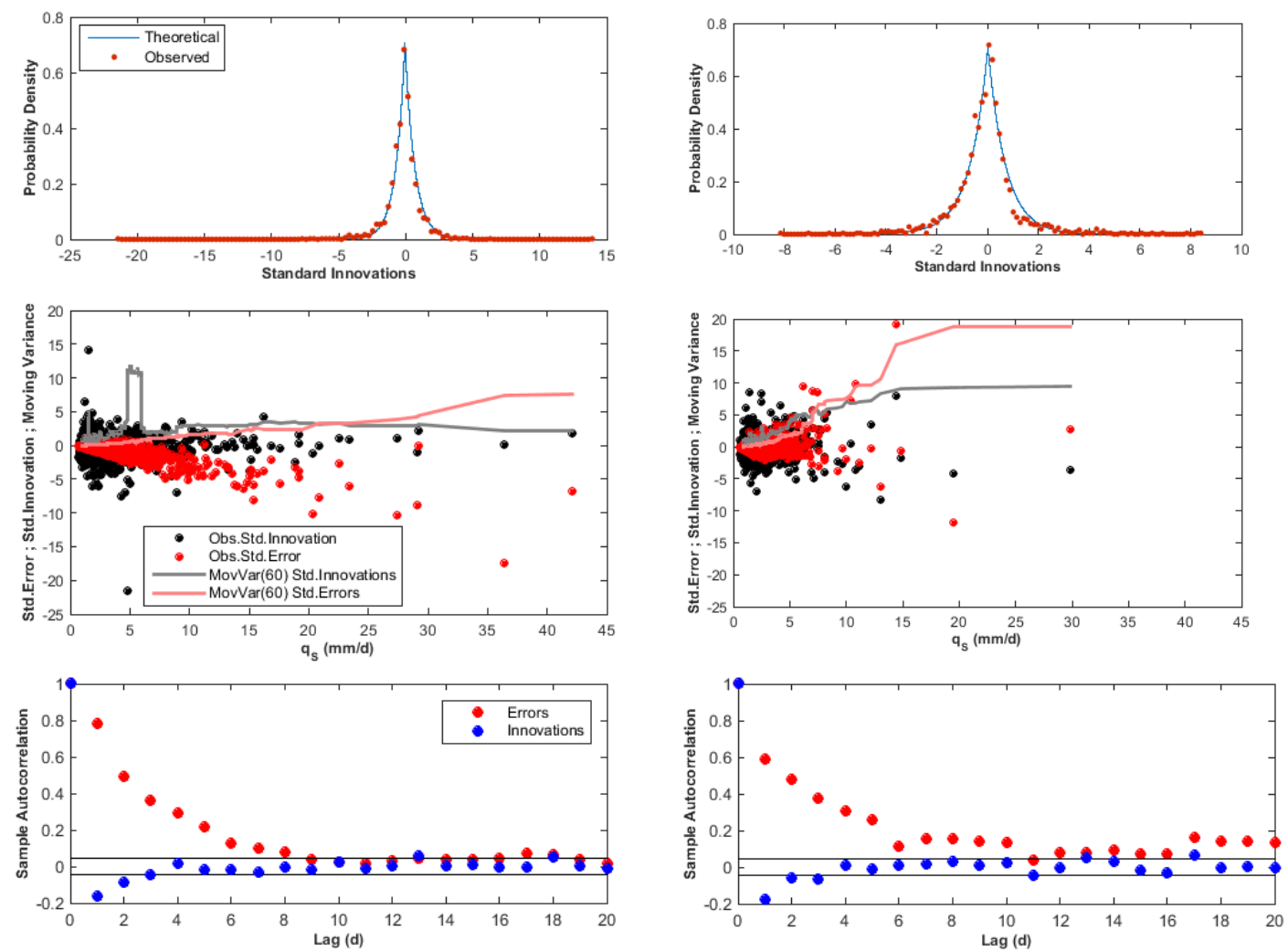

Figure 6. Test of GL++ error model hypotheses for CRR (left column) and GR4J (right column) models. In top panels, the evaluation of the fitting to the SEP distribution, of the observed standard innovations. In middle panels, the homoscedasticity evaluation for the observed standard errors (red dots and red line) and for the observed standard innovations (black dots and grey line). In bottom panels, the independence assessment for observed errors and for the innovations, through the autocorrelation function (the $95 \%$ Anderson's limits in solid black lines).

In view of a better fulfillment of the error model hypotheses (compared with SLS and WLS), it is expected that the GL++ parameter estimation could be less biased than the corresponding to those classical schemes of inference (Schoups and Vrugt, 2010). In fact, this is the reason for the poor performance shown by the biased prediction of the hydrological model: the most plausible parameter set, for both hydrological and error models, brings out (in form of a prediction bias) the deficiencies in the hydrological model and/or in the input data.

Concerning the above mentioned overpredictive bias, Table 1 shows some of its effects. There is deterioration in the NSE index and VE for the mean prediction, with respect SLS and WLS inferences, for both hydrological models. It must be also 
Hydrol. Earth Syst. Sci. Discuss., doi:10.5194/hess-2017-9, 2017

underlined the great difference between the performance of the two models which arises with GL++ inference. In contrast, with SLS and WLS inferences, the performance of both hydrological models resulted similar. The CRR performance has suffered an important deterioration, whereas the GR4J performance is still acceptable. The meaning of all these results is that GL++ inference forces a more realistic performance of the hydrological models than in SLS or WLS inferences: and this is indicating that GR4J is able to model this case study with a better performance than the CRR model. In other words, for this case study GR4J with only 4 parameters is less affected (it has a less biased mean prediction) by the structural and/or data deficiencies than the CRR model, which have 7 parameters.

The reliability assessment of the PD, through the PP-Plots in Fig. 4, shows for CRR a large overpredictive bias, much bigger than the GR4J one, confirming the previously explained mean prediction performance for both models. Furthermore, the overprediction of both hydrological models (bigger for CRR) can also be observed in Fig. 7, where the mean prediction (green solid lines) is more often above observations (red dots). In relation to the uncertainty assessment, PP-Plots in Fig. 4 show its correctness for both models. Besides, Fig. 7 shows the differences between CRR and GR4J in the appearance of the 95\% total uncertainty band: these differences are more visible in the streamflow peaks, where the overestimation in CRR also induces the greatest widening of this uncertainty band.
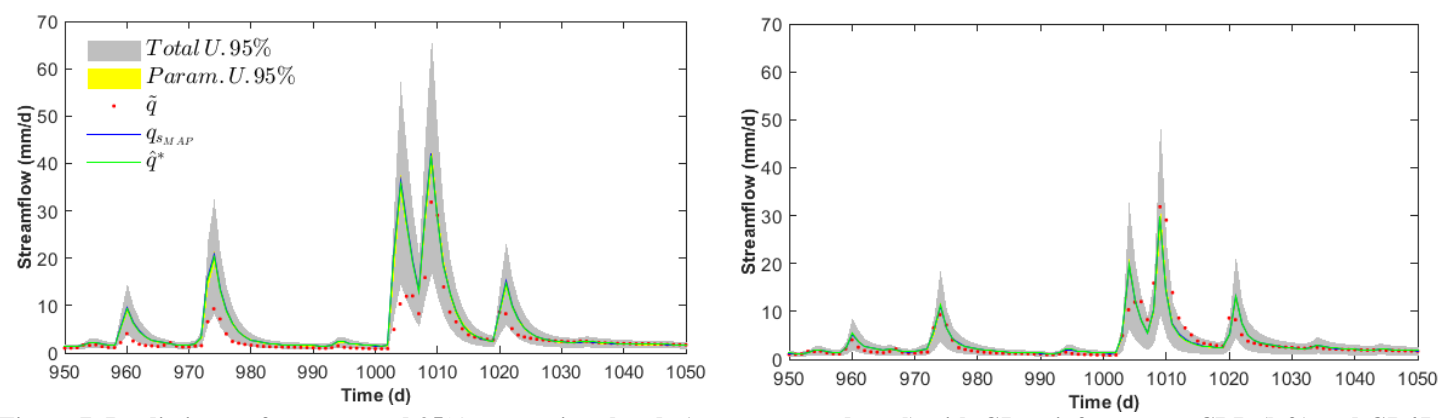

Figure 7. Predictive performance and $95 \%$ uncertainty bands (parameter and total) with GL++ inference on CRR (left) and GR4J (right) models (parameter uncertainty band is hardly appreciable).

\subsection{GL++Bias inference}

As it has been underlined, GL++ inferences introduce a bias in the PD of both hydrological models. The bias model in GL++Bias inference, which was described in section 2 by Eq. (5), considers that error bias depends on the simulated streamflow, as in the variance model, but with the flexibility of a different behavior for low and high flows. Figure 10 represents, for both CRR and GR4J hydrological models, a scatter of the errors jointly with their inferred variance and bias models. In this figure, it can be noticed the different behavior of the bias depending on the hydrological model. On the one hand, inference on CRR distinguishes (see zoomed window in Fig. 10) a different bias slope between low flows and high flows, with a threshold $q_{0}=1.96 \mathrm{~mm} / \mathrm{d}$. On the other hand, GR4J practically does not make this distinction and the inferred threshold $q_{o}=1.57 \mathrm{~mm} / \mathrm{d}$ is very close to the minimum simulated streamflow. In any case, Fig. 10 shows the good fitting of the used deterministic simple bias model: the inferred lines (blue dashed), representing the error conditional means, are placed at the central position of the errors scatter.

The inference with the GL++Bias error model yields a reasonably good fulfillment of the hypotheses about the errors. In any case, better fulfillment than previously analyzed inferences, for the case study. There are remaining problems which are described in what follows. This inference produces for both hydrological model (see Fig. 8, top panel) a pdf for the innovations with a high density for the zero and near-zero values; namely, a more kurtotic observed innovations distribution than the inferred SEP. Besides, and commonly to the previously performed GL++ inferences, the innovations autocorrelation for the first lags is not completely removed, although its values are relatively small (see Fig. 8, bottom panel). In respect to the variance stabilization, which had a poor performance in GL++, it can be observed in middle panel of Fig. 8 that the consideration of the bias model allows a good stabilization of the innovations variance, with a near-horizontal moving Page 19 of 39 
Hydrol. Earth Syst. Sci. Discuss., doi:10.5194/hess-2017-9, 2017

Manuscript under review for journal Hydrol. Earth Syst. Sci.

Published: 17 January 2017

(c) Author(s) 2017. CC-BY 3.0 License.

variance line in the CRR case, whereas for the GR4J the stabilization is also acceptable, albeit the line is not completely
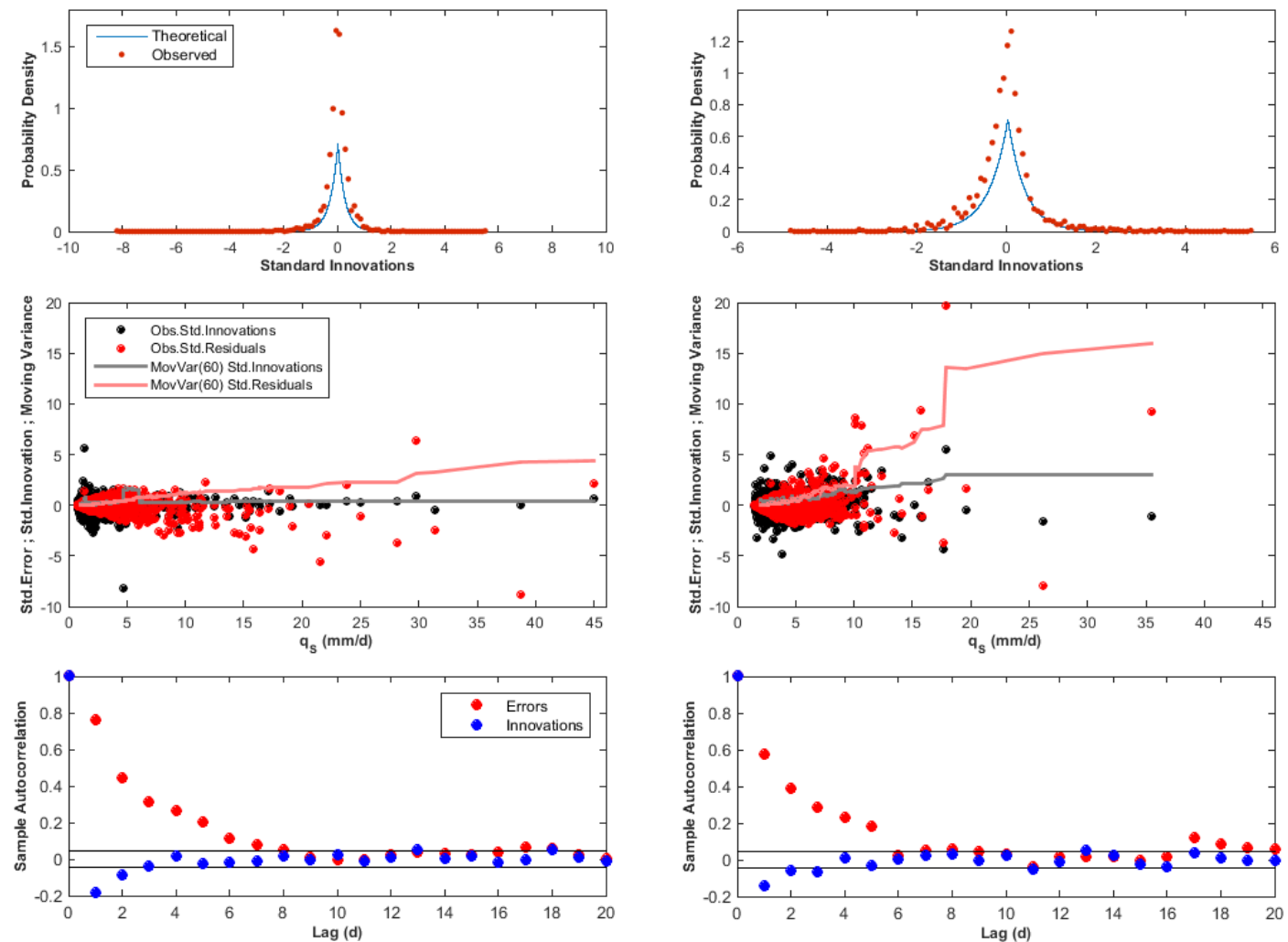

Figure 8. Test of GL++Bias error model hypotheses for CRR (left column) and GR4J (right column) models. In top panels, the evaluation of the fitting to the SEP distribution, of the observed standard innovations. In middle panels, the homoscedasticity evaluation for the observed standard errors (red dots and red line) and for the observed standard innovations (black dots and grey line). In bottom panels, the independence assessment for observed errors and for the innovations, through the autocorrelation function (the 95\% Anderson's limits in solid black lines).

The performance of the GL++Bias error model combined with the CRR hydrological model is very different from the performance with the GR4J hydrological model. As it was explained in the GL++ inference, the CRR model showed a more biased prediction than GR4J. In GL++Bias, the proposed bias model is able to considerably improve the performance of the mean prediction in CRR (green line in Fig. 9), where prediction bias is practically removed. In the case of GR4J, the bias model produces an improvement of the prediction, which is more modest: a small overpredictive bias still remains with GR4J, after the bias model inclusion.

Table 1 shows that CRR model with GL++Bias obtains for the mean prediction a NSE $=0.76$ and a VE $=0.0 \%$, whereas GR4J yields a NSE $=0.80$ and also a VE $=0.0 \%$. The null error in volume, confirms a fair performance (considered in average over all the calibration period) of the bias model. In general terms, the comparison between GL++ and GL++Bias shows that the CRR mean prediction performance has improved notably (from NSE $=0.25$ to NSE $=0.76$ ), but for GR4J the performance is nearly the same in both inferences (slightly decreasing from NSE $=0.82$ to NSE $=0.80$ ). We can conclude that the prediction bias which arises from the GR4J model with GL++ is small, but with a structure too complex to be well-reproduced by the bias model hypothesized by GL++Bias. On the contrary, most of the large bias which arises from the CRR model with GL++ can be corrected with the bias model given by GL++Bias. 
Hydrol. Earth Syst. Sci. Discuss., doi:10.5194/hess-2017-9, 2017

Manuscript under review for journal Hydrol. Earth Syst. Sci.

Published: 17 January 2017

Regarding the predictive uncertainty assessment, both GL++Bias inferences yield a reduction of the slope parameter in the variance model, in comparison with the corresponding GL++ inference (Tables 2 and 3, and black lines in Figs. 10 and 11 ). This reduction is larger for GR4J (from 0.35 to 0.15) than for CRR hydrological model (from 0.42 to 0.33). Due to this reason, the PD width for GR4J model is smaller than for CRR, which can be observed comparing the two 95\% uncertainty bands in Fig. 9. That is to say, when GL++Bias error model is used, predictions with CRR are more uncertain than with GR4J, but are also less biased. The PP-Plots (Fig. 4) exhibit the good performance of the PD for both hydrological models: even the CRR model with the GL++Bias error model shows a near-perfect fit to the 1:1 line.

Furthermore, looking at right panel of the Fig. 9, it is important to realize that the GL++Bias inference for GR4J model is the only inference that exhibits a significant contribution of parameter uncertainty to the total predictive uncertainty. This contribution seems to be underestimated in all the other performed inferences.
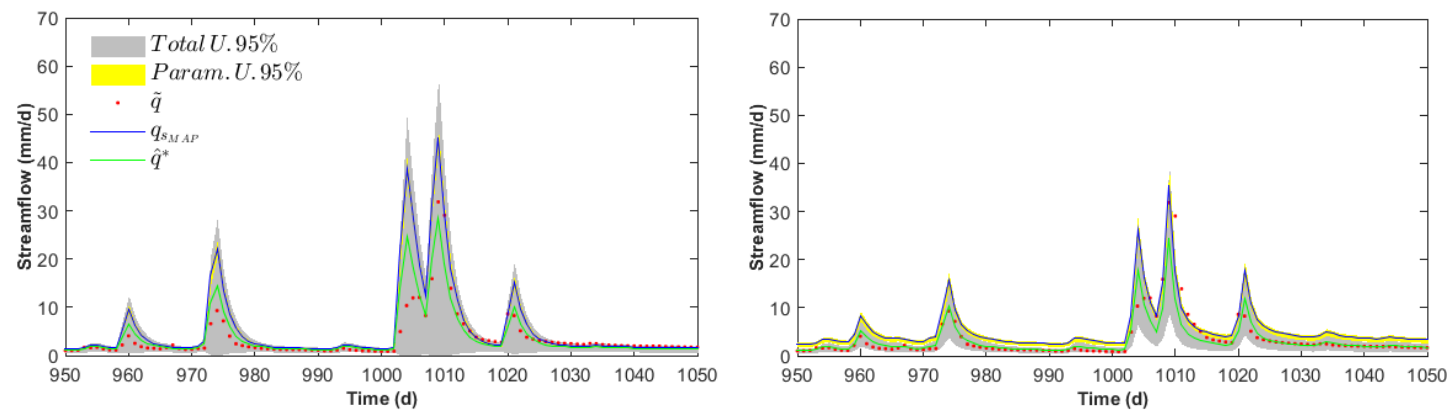

Figure 9. Predictive performance and 95\% uncertainty bands (parameter and total) with GL++Bias inference on CRR (left) and GR4J (right) hydrological models.
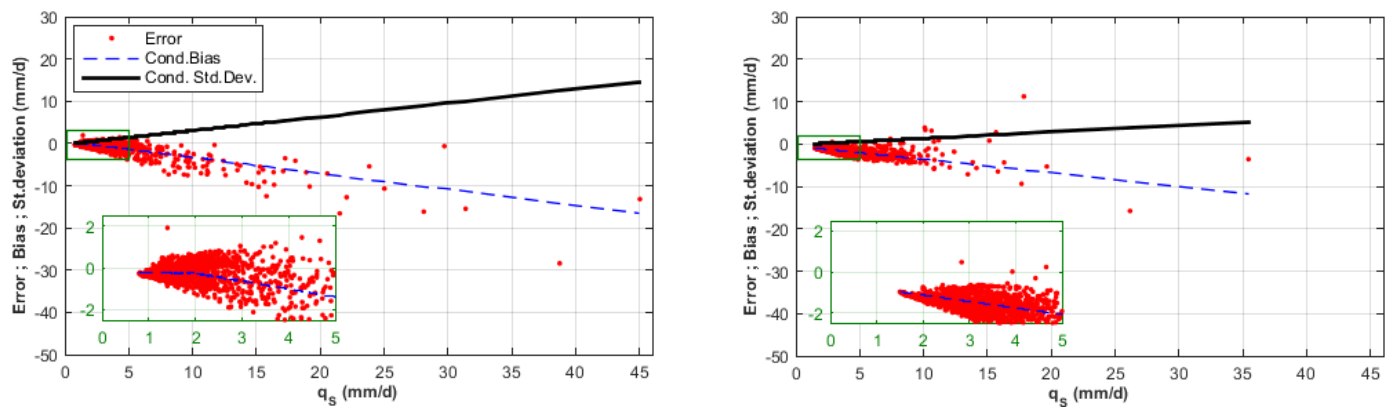

Figure 10. GL++Bias error model features for CRR (left) and GR4J (right) hydrological models: inferred conditional variance (black line) and inferred conditional bias (dashed blue line) with the error versus $q_{s}$ scatter plot. The zoom window shows the performance of the inferred bias model for low flows. 
Hydrol. Earth Syst. Sci. Discuss., doi:10.5194/hess-2017-9, 2017

\subsection{Comparison between enforcing (TL) or not enforcing (NTL) the Total Variance Law}

631

In this research, we are assuming hydrological model errors are part of a joint (bivariate) probability distribution between errors and simulated discharges at the catchment outlet. The conditional and marginal distributions belonging to a joint distribution must fulfill the TLs. As it was exposed in previous sections: i) SLS hypotheses "automatically" induce the TLs fulfillment; ii) when error statistical features are hypothesized as non-constant, and they are expressed with parametric functional forms as in Eqs. (5) and (6), the inference problem must be constrained to ensure the TLs fulfillment; iii) if the error model assumes no error bias, TEL is supposed to be "automatically" fulfilled. When TLs are not fulfilled, the inferred errors and the corresponding state variable (streamflow in this paper) cannot belong to the same bivariate joint distribution and therefore, the predictive uncertainty problem to solve is statistically incorrect.

This incorrectness generates problems, mainly related with spurious parameter interactions, affecting the inference results and making them unsuitable and possibly non-robust (Evin et al., 2014). This section will demonstrate that not enforcing the TLs is, at least, one of the most important causes of these problems. This demonstration has been done for the WLS and GL++ error models comparing the results with and without (NTL) the TLs enforcement. Since these two error models assume no error bias, only TVL must be enforced in the joint inference.

In the WLS comparison, we have found that results with and without TVL enforcement are very similar. This occurs with the two used hydrological models. The inferred hydrological parameters result similar (see Tables 2 and 3), so the hydrological model behavior is practically the same and the likelihoods are also similar (not shown). Concerning the error variance model parameters, they are not exactly equal, as Tables 2 and 3 show, but the difference seems to be negligible (e.g. for CRR model $\kappa=0.24$ in NTL case and $K=0.27$ in TL case). Therefore, it seems that WLS inference would also be able to fulfill the TLs (as SLS), without the need of enforce them. However, this is not the case. WLS generally will not fulfill the TVL if this is not enforced. But this is not an important issue in the case of the WLS inference, as it is explained in the following. Let $\{\alpha, \kappa\}_{\mathrm{ML}, \mathrm{TL}}$ be the set of variance model inferred parameters that fulfills both the maximum likelihood and the TLs criteria. It can be demonstrated that with WLS, any other set of parameters such that $\{\alpha, \kappa\}_{\mathrm{ML}}=\mathrm{C}\{\alpha, \kappa\}_{\mathrm{ML}, \mathrm{TL}}$, where $\mathrm{C}>0$ is a proportionality factor, will have the same maximum likelihood value, but it will not fulfill the TVL. Hence, with the WLS error model, the same hydrological parameter estimation is inferred, as well as similar uncertainty bands are obtained, using $\{\alpha, \kappa\}_{\mathrm{ML}, \mathrm{TL}}$ or using any other $\mathrm{c}\{\alpha, \kappa\}_{\mathrm{ML}, \mathrm{TL}}$. This means that WLS, in NTL case, is a bad-posed problem to estimate a unique identifiable set of variance model parameters. In the case study, for the CRR hydrological model, a proportionality factor of $\mathrm{C}=1.125$ has been obtained, as it can be checked in Table 2.

Regarding the comparison of the GL++ joint inferences, with and without the TLs enforcement, this research demonstrates that when the error model considers both the autocorrelation (AR) and the heteroscedasticity models, not enforcing the TLs has a significant effect on the result of the inference (unlike to what has been resulted for WLS error model). Figure 11 shows the error scatter plots as well as the inferred linear variance models for the GL++ inferences with and without the TVL enforcement. In these cases, the effects of not considering the TLs with GR4J (right panel) are always substantially larger than with CRR (left panel). These effects are the following: i) an increment of the slope in the linear variance model (see Fig. 11), from TL enforcement (black lines) to NTL (gray lines); ii) a change in the hydrological parameters, significant with GR4J (Table 3) and small with CRR hydrological model (Table 2); iii) an important erroneous uncertainty overestimation, as can be observed by the comparison between Figs. 7 (TL case) and 13 (NTL case), for the CRR model (left panels) and for GR4J (right panels); iv) changes in the PD or even radically different PDs, as it is shown for the GR4J model in the corresponding PP-Plot of the right panel in Fig. 4, where with GL++ there is a moderate overprediction, and with GL++NTL a strong underprediction; v) in NTL inferences it is observed a high correlation (as a spurious parameter interaction, which it does not exist when TLs are applied) between the slope parameter of the error standard deviation and the autocorrelation parameter (Fig. 12). This last feature was also reported in Evin et al. (2013, 2014). 
Hydrol. Earth Syst. Sci. Discuss., doi:10.5194/hess-2017-9, 2017

Manuscript under review for journal Hydrol. Earth Syst. Sci.

Published: 17 January 2017

(c) Author(s) 2017. CC-BY 3.0 License.
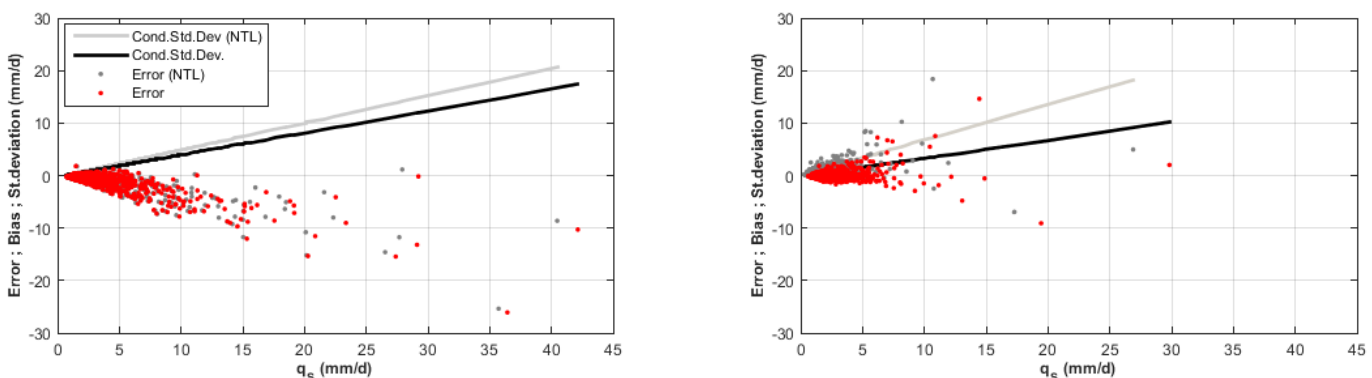

Figure 11. GL++ and GL++NTL error model features for CRR (left) and GR4J (right) hydrological models. TVL enforcement case: inferred variance model (black line) and error versus $q_{s}$ scatter (red). NTL case: inferred variance model (grey line) and error versus $\mathbf{q}_{\mathrm{s}}$ scatter (grey).

We have numerically tested in the case study the fulfillment or not of the TLs. Because WLS and GL++ assume no error bias, TVL in Eq. (7) simplifies to Eq. (21). For the CRR-GL++ inference the calculated error marginal variance value (left term in Eq. (21)) is $2.25 \mathrm{~mm}^{2} / \mathrm{d}^{2}$, which exactly matches the value for the mean of the conditional variances (right term in Eq. (21)). However, in the CRR-GL++NTL case, the calculated error marginal variance is $1.97 \mathrm{~mm}^{2} / \mathrm{d}^{2}$, which is very different from the mean of the conditional variances $\left(3.31 \mathrm{~mm}^{2} / \mathrm{d}^{2}\right)$. Therefore, in the NTL case, as it was expected, there is an imbalance between the marginal and conditional error variances.
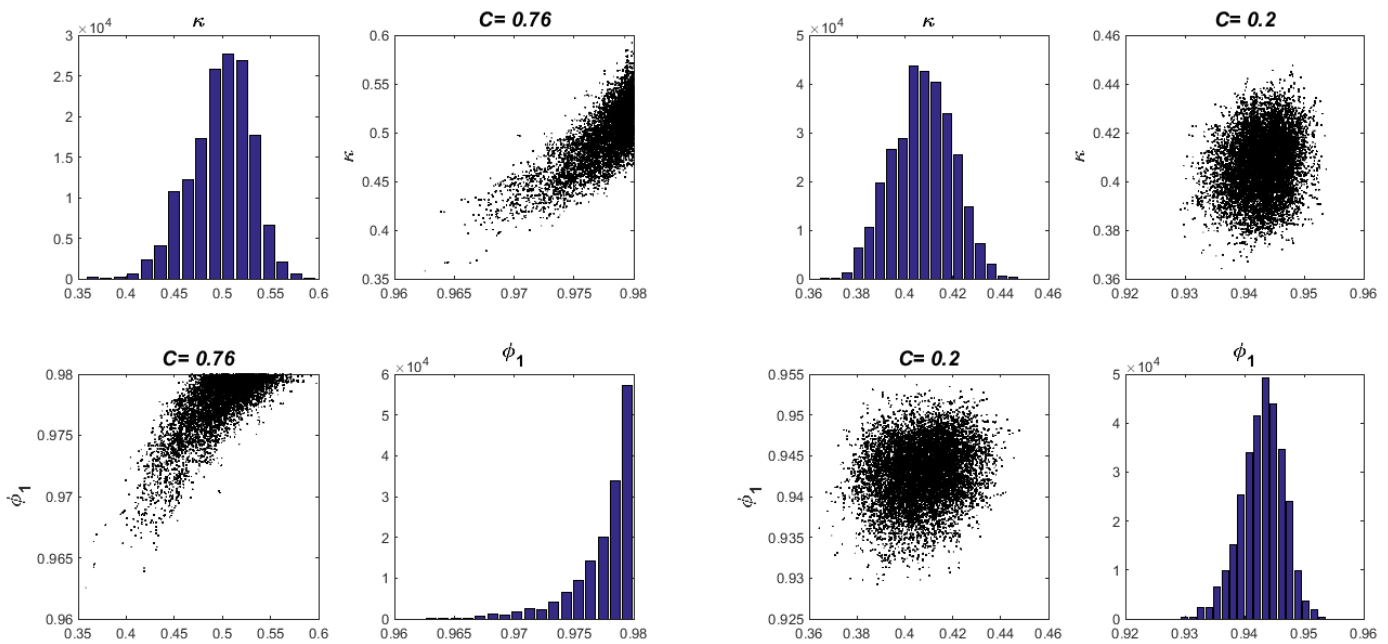
parameter of the AR(1) model and scatter plot between them, for inferences with CRR hydrological model: left panels with GL++NTL error model; right panels with GL++ error model. 

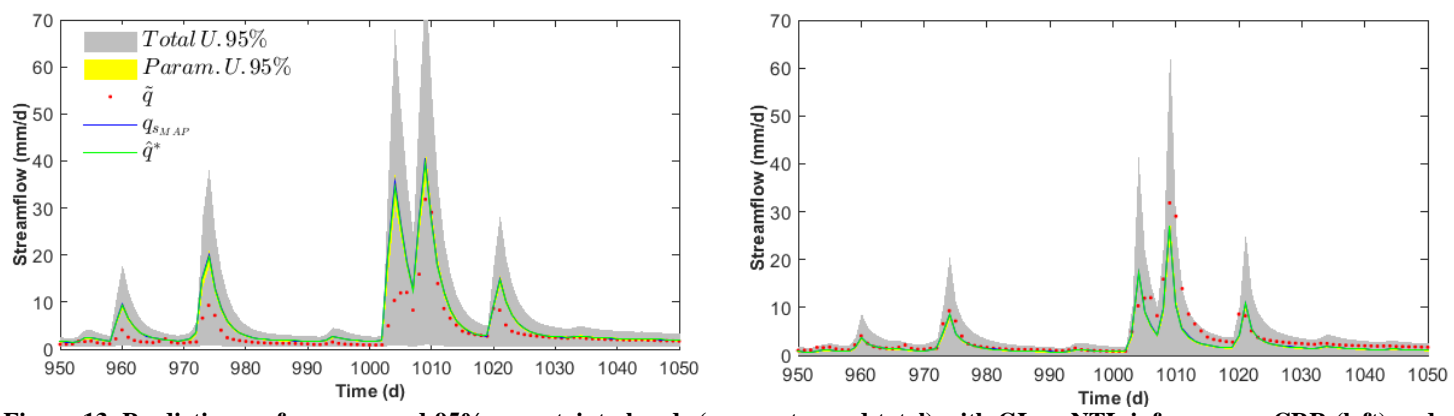

Figure 13. Predictive performance and $95 \%$ uncertainty bands (parameter and total) with GL++ NTL inference on CRR (left) and GR4J (right) models (parameter uncertainty band is hardly appreciable).

\subsection{Hydrological parameter estimates depending on the error model}

It has already stated in previous sections that an incorrect error model (actually an incorrect likelihood function) can yield apparently very good mean predictions in calibration, but with biased hydrological parameters and an incorrect uncertainty assessment. The paradigm of this idea is the SLS error model, widely used in hydrological modeling. On the contrary, a correct consideration of the errors in the inference yields more suitable parameter estimation and correct hydrological predictions, showing the model deficiencies in the form of a biased model results and an acceptable uncertainty band, even with a poor fitting to the observed data. The analyzed case study has at least a known structural problem in both models, the neglected snow processes, and for sure other problems not yet identified in the model or in the data. All these circumstances predetermine the errors structure, departing it from the SLS hypotheses of zero bias, normality, independence and homoscedasticity. Previous sections have shown how the predictions and their uncertainty evolve as a function of the hypothesized error model, from the SLS to the GL++Bias. This section will show the "other side of the coin": how the parameters change with the error model.

To illustrate the effect of the error model on the inferred parameters, it has been chosen the GR4J model, since it is parsimonious and all its four parameters have been perfectly identifiable in all performed inferences, which did not occur with the CRR model. Figure 14 presents the four parameter marginal posteriors of GR4J hydrological model when they are jointly inferred with the SLS, WLS, GL++ and GL++Bias error models. There are important differences between the marginal posterior distributions, especially for parameters $\theta_{1}, \theta_{2}$, and $\theta_{3}$. Another important characteristic to analyze is the parameter uncertainty: for parameters $\theta_{1}$ and $\theta_{2}$ it tends to increase with the more sophisticated error models, whereas for parameters $\theta_{3}$ and $\theta_{4}$ it remains similar. Concerning the corresponding CVs (Table 3), systematically SLS gives smaller values.

But the most paradigmatic and didactic proof of the necessity of making the parameter inference with a proper error model is the case of the GR4J parameter $\theta_{2}$ (the groundwater exchange coefficient). Figure 14 shows how the estimated $\theta_{2}$ suffers important changes in its value (and even in the sign!) depending on the inference. This has a deep consequence in the model conceptualization of the analyzed basin. For GR4J, a negative value of $\theta_{2}$ means that a deep (or regional) aquifer receives water from the basin. In contrast, a positive value of $\theta_{2}$ means that this regional aquifer feeds the basin with groundwater (whose origin is out of the basin). On the basis of our knowledge about the geology of FB basin, it does not have a deep aquifer with which exchange water. Hence a reasonable value for $\theta_{2}$ should be zero. I.e. the water balance of the basin must be closed without losses or contributions different to actual evapotranspiration or precipitation respectively. In absence of a regional aquifer, different values to zero are due to model and/or input deficiencies and they do not have any hydrological meaning, although they enable to close the water balance. In our case study, SLS inferred MAP value is $\theta_{2}=-0.71 \mathrm{~mm}$, WLS MAP value is still more negative, whereas with the GL++, we get a MAP value of $\theta_{2}=-0.55 \mathrm{~mm}$. All three inferences without bias need dewater the basin (negative values for $\theta_{2}$ ). Nevertheless, the most plausible inferred value for $\theta_{2}$ (the closest to zero) corresponds to $\mathrm{GL}++$, the most correct among these three error models. 
Regarding GL++Bias inference, its MAP value is $\theta_{2}=2.89 \mathrm{~mm}$. According to the previously exposed, in our case study this is an implausible value for this parameter (it is the most different from zero). Another important feature to note is that the uncertainty of $\theta_{2}$ with GL++Bias is the highest of all hydrological parameters and performed inferences, as it is shown in Fig. 14 and reflected in the CV values for this parameter in Table 3. This high uncertainty is the main responsible of the thick predictive uncertainty band due to the parameters, which was shown in Fig. 9 (right panel). In short, neglecting the bias modeling or considering an incorrect error bias model produces a biased estimation of parameter $\theta_{2}$. In general, incorrect error models will produce biased parameter estimates.
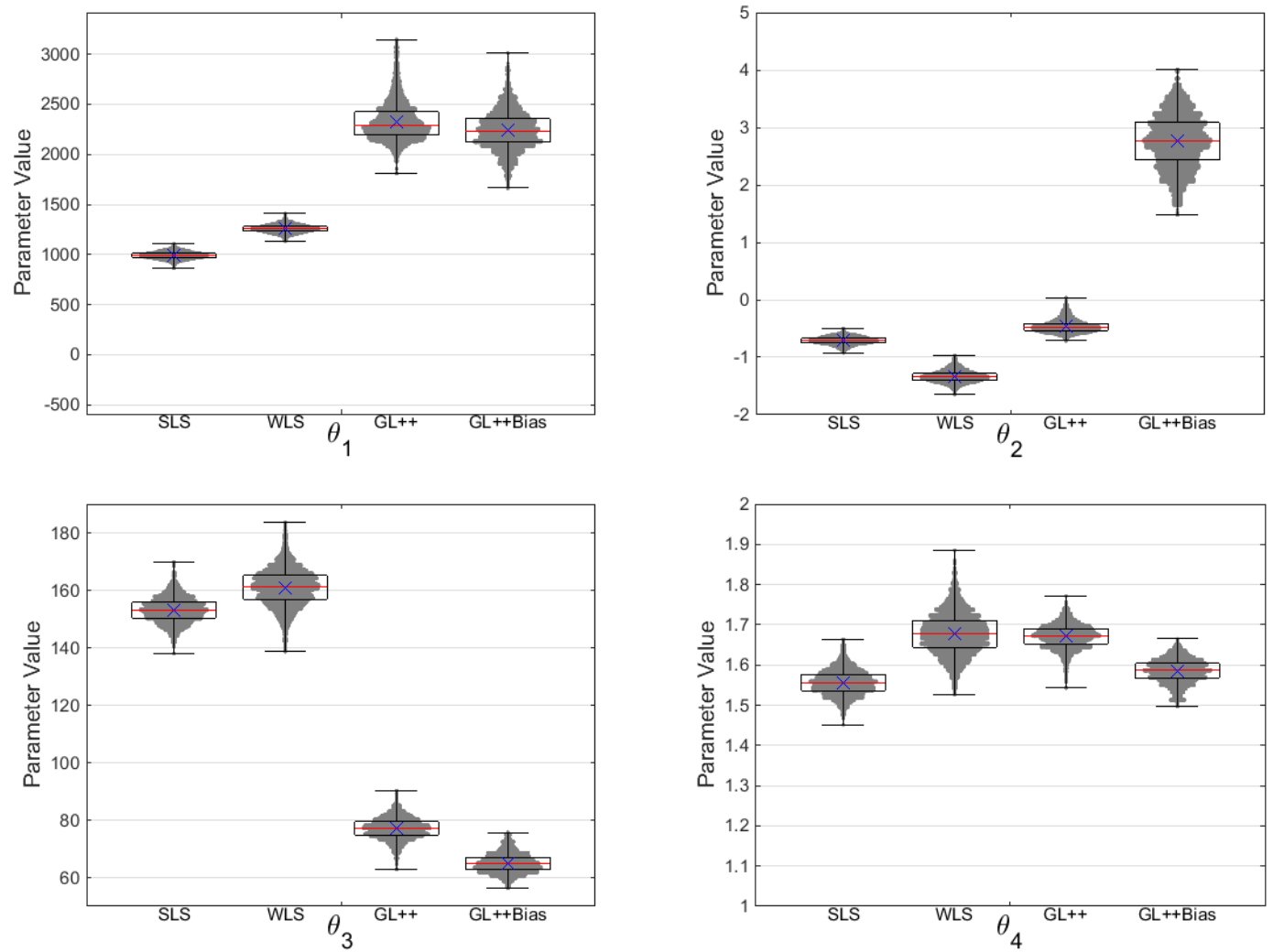

Figure 14. Evolution of the posterior distribution (box-plots) of the four GR4J model parameters with the four hypothesized error models.

Performance of the different inferences

There are several criteria in which we could rely to state why or why not an inference should be considered as a suitable inference, given a hydrological model. The first general criterion should be the degree of fulfillment of each error model hypotheses. In other words, assessing to which extent the inferred errors are unbiased, homoscedastic and independent, and also show a good fitting to the hypothesized distribution. This analysis has been exhaustively done in section 5 .

A second general criterion (which could be included into the previous one) is how accurate are the hypothesized error bias and variance models. This accuracy can be easily checked by testing if the inferred standardized errors $\eta$, defined in Eq. (8), have zero mean and unit variance. The mean and standard deviation of the standardized errors ( $\bar{\eta}$ and $S_{\eta}$ respectively) are 
shown in Table 1 for the case study. This criterion is focusing on the goodness of the error standardization (hereinafter GoES). The meaning of getting other different values to the theoretical ones (zero or one respectively) is that we are shifting and scaling the errors but not making their correct standardization; namely, the hypotheses for the error bias and/or variance models are unsuitable. It is important to realize that the fulfillment of both TLs is a necessary but not sufficient condition to obtain standardized errors with statistics satisfying or near-satisfying their theoretical values: i.e. the suitability of the error model components is also a necessary condition. Previous works (Evin et al., 2013, 2014; Scharnagl et al., 2015), where TLs were not enforced, considered that the standardized errors variance $S_{\eta}^{2}$ is, in any case, equal to one. However, inferred $S_{\eta}^{2}$ can be very different from the unit when TLs are far to be fulfilled. As an example with the GR4J hydrological model, this research got a value of $S_{\eta}=0.2$ for the GL++NTL inference, instead of the more correct value of $S_{\eta}=0.95$ for the corresponding GL++ inference (see Table 1).

The third general criterion to distinguish a fair inference is based on the general reliability of the PD. This can be visually assessed by means of the PP-Plots (as was done in section 5). Moreover the reliability of the PD can be quantified through the reliability and the resolution (or degree of certainty) indexes, defined as in Renard et al. (2010) and shown in Table 1. Generally, an inference with a proper GoES also has high reliability indexes (as occurs with the CRR-GL++Bias inference), but the opposite is not necessarily true (as occurs with the GR4J-GL++Bias inference). Given several inferences with a similar PD reliability index, those with the lowest prediction uncertainty, namely with the highest resolution, are preferable.

Finally, as the fourth general criterion, we should check and discard those inferences which exhibit parameter values whose meaning may not correspond to the knowledge we have about the basin behavior. This was already analyzed for one hydrological model in section 6.2.

Therefore, only when all previous criteria are acceptably satisfied, we could pay attention to the performance of the expected prediction (tested by its NSE and VE indexes shown in Table 1). That is to say, prediction performance of the hydrological model is a consequence (rather than a selection criterion) and it should be subordinated to the fulfillment of all previously exposed criteria. It is important to realize that, in this way, it will be obtained a set of parameter estimates which are expected to be more robust than those obtained with the classical least-squared based optimization methods.

These criteria have been applied to all the implemented inferences. Regarding SLS, the conclusion is direct: fails to meet the most of the requirements, but it has the useless property of having generally good performances of the expected prediction in calibration. Concerning WLS, this error model only fulfills with the error homoscedasticity hypothesis, and generally also exhibits good reliabilities of the PD in calibration. However, since WLS does not remove the errors serial dependence structure, it cannot be considered a correct error model when this problem appears. With respect to the GL++ and GL++Bias error models, conclusions are different depending on the hydrological model applied in the joint inference. But, a common behavior for these inferences is an acceptable fulfillment of the error model hypotheses, which is an important advance over the classical SLS and WLS error models.

To complete the discussion about both GL++ and GL++Bias error models, we will focus on Fig. 15. This figure shows a reliability-resolution diagram where are represented all inferences. The reliability-resolution diagram is useful for comparing several inferences. It is important to note that the limits between classification zones in the diagram are subjective, based on our experience, and they are provided only for guidance. The lines in the diagram are the trajectories from the simplest to the more advanced error model, starting with SLS as the reference inference. Furthermore, the NTL inferences where TLs have not been enforced are also represented with red trajectories. The main aspects to be considered in relation with Fig. 15 are the following:

i) As it has already been exposed, this diagram shows how WLS improves both reliability and resolution in relation to SLS, yielding a very good PD performance in calibration. Figure 15 also shows how WLS inferences yield similar points in the diagram between the NTL and TL cases, as theoretically expected (see section 6.1).

ii) GL++ inferences deteriorate the PD reliability in comparison with WLS. The evolution from WLS to GL++, which involves the application of an autoregressive model on the errors, is able to "convert" any of the 
Hydrol. Earth Syst. Sci. Discuss., doi:10.5194/hess-2017-9, 2017

Manuscript under review for journal Hydrol. Earth Syst. Sci.

Published: 17 January 2017

(c) Author(s) 2017. CC-BY 3.0 License.

parameter bias yielded by WLS into a bias in the prediction, but with the worsening of the PD reliability. It is expected that, the more the PD reliability difference between WLS and GL++ is, the more the parameter bias in WLS and the prediction bias in the GL++ are. According to this, it can be noticed (Fig. 15) that distance between WLS and GL++ in GR4J is much smaller than in CRR, indicating that GR4J has less structural deficiencies than CRR. I.e. GR4J is a better model for this case study.

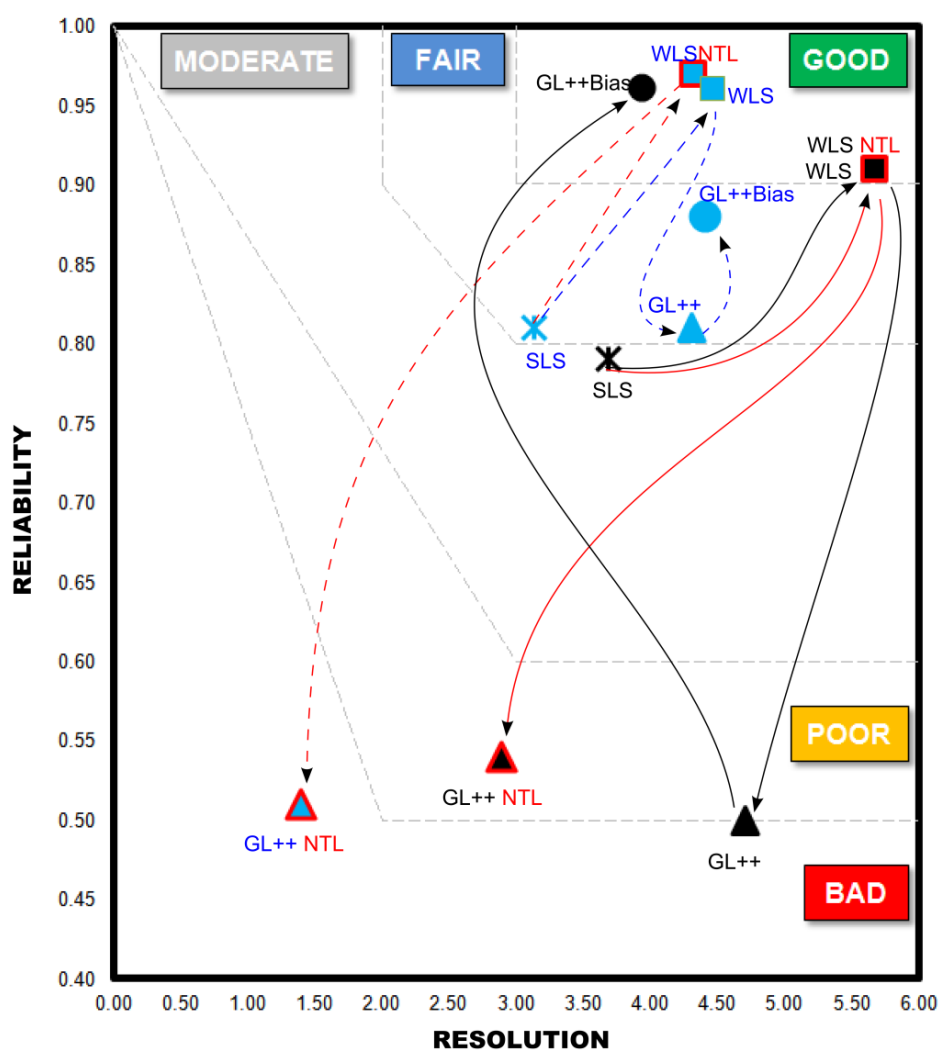

Figure 15. Reliability-Resolution diagram. Comparison of the PD performance for all inferences with the TLs enforcement: with CRR (continuous black trajectories) and with GR4J (dashed blue trajectories). Consequences on the PD when TLs are not enforced (NTL cases): with CRR model (continuous red trajectories) and with GR4J model (dashed red trajectories).

iii) The enforcement of TLs on GL++ inferences improves the performance of the PD in resolution and/or reliability in respect to the corresponding GL++NTL inferences. In our case study and for the CRR model, the improvement is mainly on the resolution, whereas with the GR4J model the inference with the enforcement of TLs yields a great improvement on both resolution and reliability indicators. In fact, for both hydrologic models, we can see that the GL++NTL inferences show a PD performance even worse (in both resolution and reliability) than the corresponding SLS inferences.

iv) The addition of a bias model to GL++ corrects in some degree the worsening in the reliability produced in the transition from WLS to GL++ for both hydrological models. The achieved reliability in the GL++Bias inference depends on the suitability of all the hypothesized error model components (bias, variance and dependence models) to reproduce the complexity of the error structure. The second criterion could be a good benchmark to test this suitability. So, for the CRR model, the GL++Bias inference yields the best reliability (see the good 
fitting to the diagonal of the PP-Plot in Fig. 4) and it also produces the best GoES statistics, as shown in Table 1. Therefore, the GL++bias inference seems to be the most suitable for the CRR model. Focusing on the GR4J model, GL++Bias inference slightly improves the reliability and the resolution, in relation to the corresponding GL++ inference. However, GL++Bias obtains GoES statistics ( $S_{\eta}=1.5$ and $\bar{\eta}=-0.28$ ) which are moderately worse than for the GL++ inference ( $S_{\eta}=0.95$ and $\bar{\eta}=-0.26$ ). We can conclude that the inferred simple error bias model is not as suitable for GR4J as it seems to be for CRR.

\section{Conclusions}

This paper has addressed the challenging problem of jointly estimate hydrological and error model parameters in a Bayesian framework, trying to solve some of the problems found in previous related researches, as in the second case study of Schoups and Vrugt (2010) as well as in Evin et al. (2014), among others.

Firstly, we have developed a new general formal likelihood function for parameters and predictive inference of hydrological models with correlated, biased, heteroscedastic, and/or non-Gaussian errors, which is based on the general likelihood function developed in Schoups and Vrugt (2010), but with the methodological modifications proposed by Evin et al. (2013).

Secondly, the detection of pitfalls in the joint inference methodology was not completed with the Evin et al. (2013) paper. In that paper and all previous related ones, the non-constant error variance and the error serial dependence were jointly modeled without considering that the errors and the simulated streamflow conform a joint distribution $p\left(e, q_{s}\right)$. Therefore, they also did not consider any relationship between the error conditional (on $q_{s}$ ) distributions and their associated error marginal distribution. The main finding of this paper is that a joint inference, to be correct, must take into account the joint distribution $p\left(e, q_{s}\right)$ and consequently the relationship between its marginal and conditional distributions. This relation is defined by two general statistical expressions called the Total Laws (TLs): the Total Expectation and the Total Variance Laws. An important consequence of the TLs enforcement is the reduction of the degrees of freedom in the inference problem: namely, the reduction of the parameter space dimension. For the case of considering more than one state variable of interest, any joint inference must take into account the joint probability distribution of all variables to be predicted and its corresponding errors. This hyper-joint distribution must also fulfill TLs.

In the presented case study, the Bayesian joint inference has been performed through the application of several inference models, as the known SLS or WLS and the new GL++ and GL++Bias. The Differential Evolution Adaptive Metropolis algorithm Dream-ZS (Schoups and Vrugt, 2010; Laloy and Vrugt, 2012) has been used to sample the posterior distribution of the hydrological and error model parameters. The inferences were carried out on two lumped hydrological models (the CRR model and the GR4J model) which were forced with daily hydrometeorological data from a basin of the MOPEX project: the French Broad basin, a wet basin of $2448 \mathrm{~km}^{2}$. The main conclusions derived from the case study analysis are summarized in the following paragraphs.

Surplus non-random information is accumulated in the errors when a hydrological model is not able to process the inputs in a correct way to reproduce the state variable observations. This model incapacity is due to deficiencies (misspecifications) in the model structure and/or in the observed input/output data reliability. The non-random errors show several symptoms (e.g. marginal and/or conditional non-zero-mean distributions, autocorrelation) which alert us about the presence of problems in the model and/or data. In presence of these problems and, if we apply the "true" but generally unknown parameter set, hydrological model simulations could be far from the observed values. Having this in mind, it is important to note that only when the jointly inferred errors fulfill the hypotheses of the error model, the hydrological model shows the most realistic prediction and the less biased parameter estimates. If we do not want or cannot improve the hydrological model and data reliability, the error model must take into account (in an aggregated way) all these deficiencies. That is the reason why GL++ inference has brought to light the deficiencies of both hydrological models, as a bias in the prediction. Moreover, GL++ has enabled to determine that, for the case study, GR4J is a better model (because shows a less biased prediction) than CRR, 
Hydrol. Earth Syst. Sci. Discuss., doi:10.5194/hess-2017-9, 2017

whereas the results of the SLS inference could suggest the opposite. In short, structural or data problems necessarily undermine either the reliability of the inferred parameters or the performance of the model results, depending on the chosen error model.

The non-fulfillment of the TLs is statistically incorrect when we are modeling a joint pdf through the definition of its conditional distributions. Only simple error models, as SLS, do not explicitly need the TLs implementation. Non-fulfillment of TLs produces incorrect parameter estimates and little reliable predictive distributions, as it was shown in NTL cases. Probably this is also the main cause of the spurious parameter interactions found in Evin et al. (2014) and shown in Fig. 12 for our case study. However, the TLs enforcement is a necessary but not sufficient condition for the correct parameter estimation and predictive uncertainty assessment: a misspecification or incorrect hypothesis about the errors statistical features can also yield biased estimates and little reliable predictive distributions, as it was also reported by Reichert and Schuwirth (2012). For example, we have demonstrated that ignoring the modeling of the error biases, when they exist, produces biased predictions. This has been shown with the GL++ inferences yielding biased predictions with both hydrological models. Besides, ignoring the bias or modeling it incorrectly produces biased parameters regarding the "true" parameter set. This was clearly shown for the parameter $\theta_{2}$ of the GR4J model.

A Bayesian formal approach to address the inference of hydrological models, as proposed in this paper, breaks away with the paradigm of parameter estimation methods based on minimizing summary statistics of model errors. In this context, this research has followed several selection criteria which are useful to discard or accept different inferences. As summary and in sequential order: i) the fulfillment of the error model hypotheses, including the adequacy of the bias and variance models to the inferred errors; ii) the general reliability of the PD; and iii) the plausibility of the parameter values in the context of the hydrological knowledge about the modeled basin. However, given the hydrological model, the resulting performance of the prediction, the reliability of its predictive uncertainty, as well as the robustness of the parameter estimates, will be exclusively conditioned by the first criterion: the degree in which errors fulfill the error model hypotheses.

\section{Acknowledgments}

This research has received funding from the Spanish Ministry of Economy and Competitiveness through the research projects SCARCE CONSOLIDER (CSD2009-00065), ECO-TETIS (CGL2011-28776-C02-01) and TETISMED (CGL2014-58127C3-3-R). We also wish to acknowledge Dr. Ezio Todini and Dr. Jasper Vrugt for constructive feedback that has helped to improve the manuscript. Dr. Vrugt is also acknowledged, for providing the code for the DREAM-ZS algorithm. The data used in this research can be downloaded from ftp://hydrology.nws.noaa.gov/pub/gcip/mopex/US Data/.

\section{Appendix A}

This appendix shows how to obtain the general likelihood function given by Eq. (17). This new likelihood function evolves from the original version in Schoups and Vrugt (2010), implementing the methodological correction proposed in Evin et al. (2013) according to which errors must be studentized before applying an autoregressive error model on them.

From the viewpoint of Bayesian statistics, not only variable observations but also model parameters are considered as random variables. Therefore, a density function of multivariate joint probability (hyper-surface) with the observations and the parameters $p\left(\tilde{y},\left\{\boldsymbol{\theta}_{h}, \boldsymbol{\theta}_{e}\right\}\right)$ must be taken into account. If, on the generated hyper-surface, a cut is made by a hyper-plane that passes through a given vector of parameters, a so-called sampling distribution (or data distribution) $p\left(\tilde{y} \mid\left\{\boldsymbol{\theta}_{h}, \boldsymbol{\theta}_{e}\right\}\right)$ is obtained, which gives the probability of the set of observations, $\tilde{y}$, conditioned on the values of this parameter vector. But when the cutting of the hyper-surface is made with a hyper-plane passing through the observations of the random variable, the concept of the likelihood function of the parameters conditioned on the observations $\ell\left(\left\{\boldsymbol{\theta}_{h}, \boldsymbol{\theta}_{e}\right\} \mid \tilde{y}\right)$ arises. The analytical expression of the likelihood is the same as for the data distribution. 
Although the likelihood function is not a probability function, the probability of a set of model parameters, given the observations, is proportional to the likelihood of these parameters. In this paper, the hypothesized error model is additive and given by:

$$
\varepsilon=y-y_{s}-\mu_{e \mid y_{s}}=e-\mu_{e \mid y_{s}}
$$

and, when an additive error model is considered, we can write:

$$
p\left(y \mid\left\{\boldsymbol{\theta}_{h}, \boldsymbol{\theta}_{e}\right\}\right)=p\left(\varepsilon \mid\left\{\boldsymbol{\theta}_{h}, \boldsymbol{\theta}_{e}\right\}\right)=\ell\left(\left\{\boldsymbol{\theta}_{h}, \boldsymbol{\theta}_{e}\right\} \mid \varepsilon\right)
$$

The Conditional Probability Law establishes the following relation among the joint, marginal and conditional probabilities of any two random variables, $\varepsilon_{1}$, and $\varepsilon_{2}$, which are conditioned to a parameter vector $\boldsymbol{\theta}$ :

$$
p\left(\varepsilon_{1}, \varepsilon_{2} \mid \boldsymbol{\theta}\right)=p\left(\varepsilon_{1} \mid \boldsymbol{\theta}\right) p\left(\varepsilon_{2} \mid \varepsilon_{1}, \boldsymbol{\theta}\right)
$$

For convenience, we can split the series of random errors, $\varepsilon=\left\{\varepsilon_{n} ; n=1 \ldots N\right\}^{T}$, into two vectors, and based on Eq. (A3) we can write:

$$
p\left(\varepsilon \mid\left\{\boldsymbol{\theta}_{h}, \boldsymbol{\theta}_{e}\right\}\right)=p\left(\varepsilon_{1} \mid\left\{\boldsymbol{\theta}_{h}, \boldsymbol{\theta}_{e}\right\}\right) p\left(\varepsilon_{2: N} \mid \varepsilon_{1},\left\{\boldsymbol{\theta}_{h}, \boldsymbol{\theta}_{e}\right\}\right)
$$

In the application of the error model defined in section 2.2, two transformations on the random errors, $\varepsilon$, were made to convert them first to the studentized errors, $\boldsymbol{\eta}$, and finally into the standardized innovations, $\boldsymbol{a}$. The same transformations must be performed on Eq. (A4) so that:

$$
p\left(\varepsilon_{1} \mid\left\{\boldsymbol{\theta}_{h}, \boldsymbol{\theta}_{e}\right\}\right)=\left|\frac{\partial \eta_{1}}{\partial \varepsilon_{1}}\right| p\left(\eta_{1} \mid\left\{\boldsymbol{\theta}_{h}, \boldsymbol{\theta}_{e}\right\}\right)=\sigma_{\varepsilon \mid q_{s(t=1)}^{-1}}^{-1} p\left(\eta_{1} \mid\left\{\boldsymbol{\theta}_{h}, \boldsymbol{\theta}_{e}\right\}\right)
$$

$$
p\left(\varepsilon_{2: N} \mid \varepsilon_{1},\left\{\boldsymbol{\theta}_{h}, \boldsymbol{\theta}_{e}\right\}\right)=\operatorname{det}|\mathbf{J}| p\left(\eta_{2: N} \mid \eta_{1},\left\{\boldsymbol{\theta}_{h}, \boldsymbol{\theta}_{e}\right\}\right)=p\left(\eta_{2: N} \mid \eta_{1},\left\{\boldsymbol{\theta}_{h}, \boldsymbol{\theta}_{e}\right\}\right) \prod_{t=2}^{N}\left|\frac{\partial \eta}{\partial \varepsilon}\right|=
$$

$$
p\left(\eta_{2: N} \mid \eta_{1},\left\{\boldsymbol{\theta}_{h}, \boldsymbol{\theta}_{e}\right\}\right) \prod_{t=2}^{N} \sigma_{\varepsilon \mid y_{s}}^{-1}=\prod_{t=2}^{N} p\left(\eta \mid \eta_{1:(t-1)},\left\{\boldsymbol{\theta}_{h}, \boldsymbol{\theta}_{e}\right\}\right) \prod_{t=2}^{N} \sigma_{\varepsilon \mid y_{s}}^{-1}=\prod_{t=2}^{N} \sigma_{\varepsilon \mid y_{s}}^{-1} p\left(\eta \mid \eta_{1:(t-1)},\left\{\boldsymbol{\theta}_{h}, \boldsymbol{\theta}_{e}\right\}\right)
$$

In Eq. (A6), $\operatorname{det}|\mathbf{J}|$ is the absolute value of the Jacobian matrix determinant for the transformation in Eq. (8). Substituting Eqs. (A5) and (A6) into Eq. (A4) we can write:

$$
p\left(\varepsilon \mid\left\{\boldsymbol{\theta}_{h}, \boldsymbol{\theta}_{e}\right\}\right)=\sigma_{\varepsilon \mid y_{s(t=1)}}^{-1} p\left(\eta_{1} \mid\left\{\boldsymbol{\theta}_{h}, \boldsymbol{\theta}_{e}\right\}\right) \prod_{t=2}^{N} \sigma_{\varepsilon \mid y_{s}}^{-1} p\left(\eta \mid \eta_{1:(t-1)},\left\{\boldsymbol{\theta}_{h}, \boldsymbol{\theta}_{e}\right\}\right)
$$

By taking into consideration Eq. (9), we can write:

$$
p\left(\varepsilon \mid\left\{\boldsymbol{\theta}_{h}, \boldsymbol{\theta}_{e}\right\}\right)=\sigma_{\varepsilon \mid y_{s(t=1)}}^{-1} p\left(\eta_{1} \mid\left\{\boldsymbol{\theta}_{h}, \boldsymbol{\theta}_{e}\right\}\right) \prod_{t=2}^{N} \sigma_{\varepsilon \mid y_{s}}^{-1} p\left(\eta \mid \eta_{(t-1)},\left\{\boldsymbol{\theta}_{h}, \boldsymbol{\theta}_{e}\right\}\right)=
$$

$$
\sigma_{\varepsilon \mid y_{s(t=1)}}^{-1} p\left(\eta_{1} \mid\left\{\boldsymbol{\theta}_{h}, \boldsymbol{\theta}_{e}\right\}\right) \prod_{t=2}^{N} \sigma_{\varepsilon \mid y_{s}}^{-1} p\left(z \mid\left\{\boldsymbol{\theta}_{h}, \boldsymbol{\theta}_{e}\right\}\right)
$$


Using the approximation for $t=1$ given by Schoups and Vrugt (2010), Eq. (A8) can be rewritten as:

$$
p\left(\varepsilon \mid\left\{\boldsymbol{\theta}_{h}, \boldsymbol{\theta}_{e}\right\}\right) \cong \prod_{t=1}^{N} \sigma_{\varepsilon \mid y_{s}}^{-1} p\left(z \mid\left\{\boldsymbol{\theta}_{h}, \boldsymbol{\theta}_{e}\right\}\right)
$$

Finally, if we apply the transformation performed in Eq. (11) to Eq. (A9), we obtain the final expression of the likelihood function:

$$
\ell\left(\left\{\boldsymbol{\theta}_{h}, \boldsymbol{\theta}_{e}\right\} \mid \varepsilon\right)=p\left(\varepsilon \mid\left\{\boldsymbol{\theta}_{h}, \boldsymbol{\theta}_{e}\right\}\right) \cong \prod_{t=1}^{N} \sigma_{\varepsilon \mid y_{s}}^{-1} \sigma_{\mathbf{z}}^{-1} p\left(a \mid\left\{\boldsymbol{\theta}_{h}, \boldsymbol{\theta}_{e}\right\}\right)
$$

Since we have assumed in section 2.2 that the innovations, $\boldsymbol{a}$, must fit a Skew Exponential Power distribution (SEP), term $p\left(a \mid\left\{\boldsymbol{\theta}_{h}, \boldsymbol{\theta}_{e}\right\}\right)$ is assessed by Eq. (13). To facilitate numerical computation, it is common to use the log-likelihood function. By taking logarithms on Eq. (A10), the resulting expression is:

$$
\mathcal{L}\left(\left\{\boldsymbol{\theta}_{h}, \boldsymbol{\theta}_{e}\right\} \mid \varepsilon\right) \cong N \log \frac{2 \sigma_{\xi} w_{\beta}}{\sigma_{\mathbf{z}}\left(\xi+\xi^{-1}\right)}-\sum_{t=1}^{N} \log \sigma_{\varepsilon \mid y_{s}}-c_{\beta} \sum_{t=1}^{N}\left|a_{\xi}\right|^{\frac{2}{1+\beta}}
$$

\section{Appendix B}

The following lines explain in detail how to enforce the Total Laws in the inferences WLS, GL++ and GL++Bias. WLS and GL++ implicitly fulfill the Total Expectation Law (TEL), because they are supposed to have unbiased errors. Therefore, let's start with the Total Variance Law (TVL) which must be enforced in all cases. From the properties of the variance operator, we can write the following expression applied to an error variance model:

$$
V\left[\sigma_{e \mid q_{s}}\right]=E\left[\sigma_{e \mid q_{s}}^{2}\right]-E^{2}\left[\sigma_{e \mid q_{s}}\right]
$$

Solving for $E\left[\sigma_{e \mid q_{s}}\right]$ in the last equation we can write:

$$
E\left[\sigma_{e \mid q_{s}}\right]=\left(E\left[\sigma_{e \mid q_{s}}^{2}\right]-V\left[\sigma_{e \mid q_{s}}\right]\right)^{0.5}
$$

Considering the TVL given by Eq. (7) for a variance and bias error models, we can write:

$$
V[e]=E\left[\sigma_{e \mid q_{s}}^{2}\right]+V\left[\mu_{e \mid q_{s}}\right]
$$

and, solving for the first right term and substituting in Eq. (B2) we obtain:

$$
E\left[\sigma_{e \mid q_{s}}\right]=\left(V[e]-V\left[\mu_{e \mid q_{s}}\right]-V\left[\sigma_{e \mid q_{s}}\right]\right)^{0.5}
$$

In Eq. (B4) the expressions for $E\left[\sigma_{e \mid q_{s}}\right], V\left[\mu_{e \mid q_{s}}\right]$ and $V\left[\sigma_{e \mid q_{s}}\right]$ can be derived through the application of the expectation and variance operators on Eq. (5) for the bias model, and on Eq. (6) for the variance model. This is carried out as follows: 
Hydrol. Earth Syst. Sci. Discuss., doi:10.5194/hess-2017-9, 2017

Manuscript under review for journal Hydrol. Earth Syst. Sci.

Published: 17 January 2017

(c) Author(s) 2017. CC-BY 3.0 License.

$$
E\left[\sigma_{e \mid q_{s}}\right]=\int_{-\infty}^{+\infty} \sigma_{e \mid q_{s}} f\left(q_{s}\right) d q_{s}=\alpha+\kappa E\left[q_{s}\right]
$$

$$
V\left[\mu_{e \mid q_{s}}\right]=0 \quad \text { if } q_{s} \leq q_{0}
$$

where the expectation of the bias model is given by:

$$
\begin{array}{lll}
E\left[\mu_{e \mid q_{s}}\right]=\gamma & \text { if } & q_{s} \leq q_{0} \\
E\left[\mu_{e \mid q_{s}}\right]=\gamma-\tau q_{0}+\tau E\left[q_{s}\right] & \text { if } & q_{s}>q_{0}
\end{array}
$$

For the hypothesis of zero-mean errors, adopted by inferences WLS and GL++, our bias model is $\mu_{e \mid q_{s}}=0 \forall q_{s}$ and then $V\left[\mu_{e \mid q_{s}}\right]=0$. Therefore, substituting Eqs. (B5) and (B7) into Eq. (B4) and solving for the intercept parameter of the variance model, $\alpha$, we obtain:

$$
\alpha=\left(V[e]-\kappa^{2} V\left[q_{s}\right]\right)^{0.5}-\kappa E\left[q_{s}\right]
$$

which is the only non-independent parameter under the zero-mean error hypothesis and corresponds to the Eq. (22).

Our model GL++Bias has the hypothesis of having not zero-mean errors. In this situation, we must enforce both TVL and TEL and, as a consequence, three more error model parameters are not independent. Applying the TEL in Eq. (3) with an error model with bias, we can write:

$$
E[e]=E\left[\mu_{e \mid q_{s}}\right]
$$

Considering our bias model defined in Eq. (5) and using the results given by Eq. (B8) we obtain:

$$
\begin{aligned}
& \gamma=E[e] \quad \text { if } \quad q_{s} \leq q_{0} \\
& \gamma=E[e]+\tau q_{0}-\tau E\left[q_{s}\right] \text { if } \quad q_{s}>q_{0}
\end{aligned}
$$

where, $\gamma$ is the second non-independent parameter. First expression in Eq. (B11) corresponds to Eq. (25). If we equate both expressions in Eq. (B11), for the streamflow threshold $q_{0}$, we can obtain the expression for parameter $\tau$, which is the third non-independent parameter and corresponds to Eq. (26):

$$
\tau=\frac{E\left[e_{1}\right]-E\left[e_{2}\right]}{q_{0}-E\left[q_{s_{2}}\right]}
$$


Hydrol. Earth Syst. Sci. Discuss., doi:10.5194/hess-2017-9, 2017

Manuscript under review for journal Hydrol. Earth Syst. Sci.

Published: 17 January 2017

(c) Author(s) 2017. CC-BY 3.0 License.

948 where, subindexes 1 and 2 distinguish the low and high streamflow populations respectively. Finally, since the 949 heteroscedasticity model parameters, $\alpha$ and $\kappa$, are the same for both streamflow populations, and taking into account 950 equations (B5), (B6) and (B7) for their substitution into the Eq. (B4):

$$
\begin{aligned}
\alpha & =\left(V\left[e_{1}\right]-\kappa^{2} V\left[q_{s_{1}}\right]\right)^{0.5}-\kappa E\left[q_{s_{1}}\right]= \\
& =\left(V\left[e_{2}\right]-\left(\tau^{2}+\kappa^{2}\right) V\left[q_{s_{2}}\right]\right)^{0.5}-\kappa E\left[q_{s_{2}}\right]
\end{aligned}
$$

954

which corresponds to Eq. (24).

From Eq. (B13), $\kappa$ can be solved iteratively, and it is the fourth and last non-independent parameter. When parameter $\alpha$ is close to zero, an approximate explicit solution is given by:

$$
\kappa=\left(\frac{V\left[e_{1}\right]-V\left[e_{2}\right]+\tau^{2} V\left[q_{s_{2}}\right]}{E\left[q_{s_{1}}^{2}\right]-E\left[q_{s_{2}}^{2}\right]}\right)^{0.5} \quad \text { for } \quad \alpha \approx 0
$$


Anderson, R. L.: Distribution of the serial correlation coefficient, Ann. Math. Stat., 13(1), 1--13, doi:10.1214/aoms/1177731638, 1942.

Bates, B. C. and Campbell, E. P.: A Markov chain Monte-Carlo scheme for parameter estimation and inference in conceptual rainfall-runoff modeling, Water Resour. Res., 37(4), 937-947, doi:10.1029/2000WR900363, 2001.

Blitzstein, J. K. and Hwang, J.: Introduction to Probability, PRESS, CRC., 2014.

Cheng, Q. B., Chen, X., Xu, C. Y., Reinhardt-Imjela, C. and Schulte, A.: Improvement and comparison of likelihood 519(PB), 2202-2214, doi:10.1016/j.jhydrol.2014.10.008, 2014.

Cowles, M. K. and Carlin, B. P.: Markov chain Monte Carlo convergence diagnostics: a comparative review, J. Amer. Stat. Assoc., 91(434), 883-904, doi:10.2307/2291683, 1996.

Duan, Q., Schaake, J., Andrassian, V., Franks, S., Goteti, G., Gupta, H. V., Gusev, Y. M., Habets, F., Hall, A., Hay, L., Hogue, T., Huang, M., Leavesley, G., Liang, X., Nasonova, O. N., Noilhan, J., Oudin, L., Sorooshian, S., Wagener, T. and Wood, E. F.: Model Parameter Estimation Experiment (MOPEX): An overview of science strategy and major results from the second and third workshops, in Journal of Hydrology, vol. 320, pp. 3-17., 2006. autocorrelation in hydrological model calibration, Water Resour. Res., 49(7), 4518-4524, doi:10.1002/wrcr.20284, 2013.

Evin, G., Thyer, M., Kavetski, D., McInerney, D. and Kuczera, G.: Comparison of joint versus postprocessor approaches for hydrological uncertainty estimation accounting for error autocorrelation and heteroscedasticity, Water Resour. Res., 50(3), 2350-2375, doi:10.1002/2013WR014185, 2014.

Gelb, A.: Applied Optimal Estimation, MIT Press., 2001.

Gelman, A. and Rubin, D. B.: Inference from Iterative Simulation Using Multiple Sequences, Stat. Sci., 7(4), 457-472, doi:10.2307/2246093, 1992.

Del Giudice, D., Honti, M., Scheidegger, A., Albert, C., Reichert, P. and Rieckermann, J.: Improving uncertainty estimation in urban hydrological modeling by statistically describing bias, Hydrol. Earth Syst. Sci., 17(10), 4209-4225, doi:10.5194/hess-17-4209-2013, 2013.

Han, F. and Zheng, Y.: Multiple-response Bayesian calibration of watershed water quality models with significant input and model structure errors, Adv. Water Resour., 88, 109-123, doi:10.1016/j.advwatres.2015.12.007, 2016.

990 pp. 49-68, AGU, Washington, DC., 2002.

991 Kavetski, D., Kuczera, G. and Franks, S. W.: Bayesian analysis of input uncertainty in hydrological modeling: 1. Theory, 992 Water Resour. Res., 42(3), n/a-n/a, doi:10.1029/2005WR004368, 2006a.

993 Kavetski, D., Kuczera, G. and Franks, S. W.: Bayesian analysis of input uncertainty in hydrological modeling: 2. 994 Application, Water Resour. Res., 42(3), n/a-n/a, doi:10.1029/2005WR004376, 2006 b. 
17-31, doi:10.1007/BF02428423, 1997.

Krzysztofowicz, R.: Bayesian theory of probabilistic forecasting via deterministic hydrologic model, Water Resour. Res., 35(9), 2739-2750, doi:10.1029/1999WR900099, 1999.

Kuczera, G.: Improved parameter inference in catchment models: 1. Evaluating parameter uncertainty, Water Resour. Res., 19(5), 1151-1162, doi:10.1029/WR019i005p01151, 1983.

Kuczera, G., Kavetski, D., Franks, S. and Thyer, M.: Towards a Bayesian total error analysis of conceptual rainfall-runoff models: Characterising model error using storm-dependent parameters, J. Hydrol., 331(1-2), 161-177, doi:10.1016/j.jhydrol.2006.05.010, 2006.

Laio, F. and Tamea, S.: Verification tools for probabilistic forecasts of continuous hydrological variables, Hydrol. Earth Syst. Sci. Discuss., 3(4), 2145-2173, doi:10.5194/hessd-3-2145-2006, 2006.

Laloy, E. and Vrugt, J. A.: High-dimensional posterior exploration of hydrologic models using multiple-try DREAM (ZS) and high-performance computing, Water Resour. Res., 48(1), 1-18, doi:10.1029/2011WR010608, 2012.

Mantovan, P. and Todini, E.: Hydrological forecasting uncertainty assessment: Incoherence of the GLUE methodology, J. Hydrol., 330(1-2), 368-381, doi:10.1016/j.jhydrol.2006.04.046, 2006.

Metropolis, N., Rosenbluth, A. W., Rosenbluth, M. N., Teller, A. H. and Teller, E.: Equation of state calculations by fast computing machines, J. Chem. Phys., 21(6), 1087-1092, doi:http://dx.doi.org/10.1063/1.1699114, 1953.

Montanari, A., \& Brath, a.: A stochastic approach for assessing the uncertainty of rainfall-runoff simulations, Water Resour. Res., 40(1), 1-11, doi:10.1029/2003WR002540, 2004.

Montanari, A. and Koutsoyiannis, D.: A blueprint for process-based modeling of uncertain hydrological systems, Water Resour. Res., 48(9), n/a-n/a, doi:10.1029/2011WR011412, 2012.

Perrin, C., Michel, C. and Andréassian, V.: Improvement of a parsimonious model for streamflow simulation, J. Hydrol., 279(1-4), 275-289, doi:10.1016/S0022-1694(03)00225-7, 2003.

Pokhrel, P. and Gupta, H. V.: On the use of spatial regularization strategies to improve calibration of distributed watershed models, Water Resour. Res., 46(1), 1-17, doi:10.1029/2009WR008066, 2010.

Reichert, P. and Mieleitner, J.: Analyzing input and structural uncertainty of nonlinear dynamic models with stochastic, timedependent parameters, Water Resour. Res., 45(10), n/a--n/a, doi:10.1029/2009WR007814, 2009.

Reichert, P. and Schuwirth, N.: Linking statistical bias description to multiobjective model calibration, Water Resour. Res., 48(9), n/a--n/a, doi:10.1029/2011WR011391, 2012.

Renard, B., Kavetski, D., Kuczera, G., Thyer, M. and Franks, S. W.: Understanding predictive uncertainty in hydrologic modeling: The challenge of identifying input and structural errors, Water Resour. Res., 46(5), W05521, doi:10.1029/2009WR008328, 2010.

Renard, B., Kavetski, D., Leblois, E., Thyer, M., Kuczera, G. and Franks, S. W.: Toward a reliable decomposition of predictive uncertainty in hydrological modeling: Characterizing rainfall errors using conditional simulation, Water Resour. Res., 47(11), doi:10.1029/2011WR010643, 2011.

Sage, A. and Melsa, J.: Estimation theory with applications to communications and control, McGraw-Hill., 1971.

Scharnagl, B., Iden, S. C., Durner, W., Vereecken, H. and Herbst, M.: Inverse modelling of in situ soil water dynamics: accounting for heteroscedastic, autocorrelated, and non-Gaussian distributed residuals, Hydrol. Earth Syst. Sci. Discuss., 12(2), 2155-2199, doi:10.5194/hessd-12-2155-2015, 2015.

Schoups, G. and Vrugt, J. A.: A formal likelihood function for parameter and predictive inference of hydrologic models with Page 35 of 39 
correlated, heteroscedastic, and non-Gaussian errors, Water Resour. Res., 46(10), 1-17, doi:10.1029/2009WR008933, 2010.

Schoups, G., Vrugt, J. A., Fenicia, F. and Van De Giesen, N. C.: Corruption of accuracy and efficiency of Markov chain Monte Carlo simulation by inaccurate numerical implementation of conceptual hydrologic models, Water Resour. Res., 46(10), W10530, doi:10.1029/2009WR008648, 2010.

Smith, T., Marshall, L. and Sharma, A.: Modeling residual hydrologic errors with Bayesian inference, J. Hydrol., 528(Sept2015), 29-37, doi:10.1016/j.jhydrol.2015.05.051, 2015.

Sorooshian, S. and Dracup, J. A.: Stochastic parameter estimation procedures for hydrologie rainfall-runoff models: Correlated and heteroscedastic error cases, Water Resour. Res., 16(2), 430-442, doi:10.1029/WR016i002p00430, 1980.

Sorooshian, S. and Gupta, V. K.: Automatic calibration of conceptual rainfall-runoff models: The question of parameter observability and uniqueness, Water Resour. Res., 19(1), 260-268, doi:10.1029/WR019i001p00260, 1983.

Thyer, M., Renard, B., Kavetski, D., Kuczera, G., Franks, S. W. and Srikanthan, S.: Critical evaluation of parameter consistency and predictive uncertainty in hydrological modeling: A case study using Bayesian total error analysis, Water Resour. Res., 45(12), n/a--n/a, doi:10.1029/2008WR006825, 2009.

Todini, E.: Hydrological catchment modelling: past, present and future, Hydrol. Earth Syst. Sci., 11(1), 468-482, doi:10.5194/hess-11-468-2007, 2007.

Vrugt, J. a., ter Braak, C. J. F., Clark, M. P., Hyman, J. M. and Robinson, B. a.: Treatment of input uncertainty in hydrologic modeling: Doing hydrology backward with Markov chain Monte Carlo simulation, Water Resour. Res., 44, 1-52, doi:10.1029/2007WR006720, 2008.

Vrugt, J. a., ter Braak, C. J. F., Diks, C. G. H., Robinson, B. a., Hyman, J. M. and Higdon, D.: Accelerating Markov Chain Monte Carlo Simulation by Differential Evolution with Self-Adaptive Randomized Subspace Sampling, Int. J. Nonlinear Sci. Numer. Simul., 10(3), 273-290, doi:10.1515/IJNSNS.2009.10.3.273, 2009a.

Vrugt, J. A., ter Braak, C. J. F., Gupta, H. V. and Robinson, B. A.: Equifinality of formal (DREAM) and informal (GLUE) Bayesian approaches in hydrologic modeling?, Stoch. Environ. Res. Risk Assess., 23(7), 1011-1026, doi:10.1007/s00477008-0274-y, 2009b.

Westra, S., Thyer, M., Leonard, M., Kavetski, D. and Lambert, M.: A strategy for diagnosing and interpreting hydrological model non-stationarity, Water Resour. Res., 1-24, doi:10.1002/2013WR014719.Received, 2014.

Zheng, Y. and Han, F.: Markov Chain Monte Carlo (MCMC) uncertainty analysis for watershed water quality modeling and management, Stoch. Environ. Res. Risk Assess., 30(1), 293-308, doi:10.1007/s00477-015-1091-8, 2016. 
Hydrol. Earth Syst. Sci. Discuss., doi:10.5194/hess-2017-9, 2017

Manuscript under review for journal Hydrol. Earth Syst. Sci.

Published: 17 January 2017

(c) Author(s) 2017. CC-BY 3.0 License.

1066

\begin{tabular}{|c|c|c|c|c|c|c|c|c|}
\hline & \multicolumn{4}{|c|}{ CRR } & \multicolumn{4}{|c|}{ GR4J } \\
\hline & SLS & WLS & GL++ & GL++Bias & SLS & WLS & GL++ & GL++Bias \\
\hline Log-L & -1625.5 & -461.3 & 759.0 & 773.3 & -1819.9 & -726.1 & 742.2 & 749.9 \\
\hline NSE* & 0.90 & 0.87 & 0.25 & 0.76 & 0.87 & 0.85 & 0.82 & 0.80 \\
\hline RMSE* & 0.59 & 0.66 & 1.59 & 0.91 & 0.66 & 0.70 & 0.78 & 0.83 \\
\hline VE* (\%) & 5.5 & 2.7 & 32.9 & 0.0 & 0.8 & -3.6 & 2.8 & 0.0 \\
\hline $\mathbf{S}_{\eta}$ & --- & 0.88 & 0.76 & 1.04 & --- & 0.79 & 0.95 & 1.5 \\
\hline$\underline{\eta}$ & --- & -0.09 & -0.73 & 0.04 & --- & 0.07 & -0.26 & -0.28 \\
\hline Reliability & 0.79 & 0.91 & 0.50 & 0.96 & 0.81 & 0.96 & 0.81 & 0.88 \\
\hline Resolution & 3.68 & 5.66 & 4.70 & 3.94 & 3.13 & 4.45 & 4.30 & 4.40 \\
\hline
\end{tabular}


Hydrol. Earth Syst. Sci. Discuss., doi:10.5194/hess-2017-9, 2017

Manuscript under review for journal Hydrol. Earth Syst. Sci.

Published: 17 January 2017

(c) Author(s) 2017. CC-BY 3.0 License.
Hydrology and (c) (i)

1073

Table 2. Maximum a posteriori (MAP) and coefficient of variation (CV) of inferred parameters, for all inferences with CRR hydrological model. CVs equal or larger than 0.2 are marked in red colour. Inferred parameters by the TLs enforcement are marked in shadow.

\begin{tabular}{|c|c|c|c|c|c|c|c|c|c|c|c|c|c|}
\hline & & & & \multicolumn{4}{|c|}{ WLS } & \multicolumn{4}{|c|}{ GL++ } & \multirow{2}{*}{\multicolumn{2}{|c|}{$\frac{\text { GL++ Bias }}{\text { TL }}$}} \\
\hline & & \multicolumn{2}{|c|}{ SLS } & \multicolumn{2}{|c|}{ NTL } & \multicolumn{2}{|c|}{$\mathrm{TL}$} & \multicolumn{2}{|c|}{ NTL } & \multicolumn{2}{|c|}{$\mathrm{TL}$} & & \\
\hline & & MAP & CV & MAP & CV & MAP & CV & MAP & CV & MAP & $\mathrm{CV}$ & MAP & CV \\
\hline & Imax & 10.00 & 0.06 & 2.32 & 0.18 & 2.07 & 0.17 & 0.49 & 0.47 & 0.48 & 0.42 & 0.96 & 0.42 \\
\hline & Smax & 207 & 0.05 & 369 & 0.07 & 363 & 0.06 & 37 & 0.80 & 34 & 0.13 & 36 & 0.09 \\
\hline & Qsmax & 7.60 & 0.07 & 3.67 & 0.08 & 3.76 & 0.07 & 16 & 0.76 & 12 & 0.12 & 8.84 & 0.11 \\
\hline$\frac{\alpha}{0}$ & $\alpha_{\mathrm{e}}$ & 97 & 0.20 & 12 & 0.09 & 12 & 0.09 & 89 & 0.33 & 90 & 0.28 & 85 & 0.29 \\
\hline & $\alpha_{f}$ & -0.12 & 1.77 & -0.64 & 0.43 & -0.60 & 0.38 & -0.36 & 3.88 & -0.768 & 0.72 & -1.26 & 0.20 \\
\hline & $K_{f}$ & 2.46 & 0.02 & 3.14 & 0.03 & 3.15 & 0.03 & 1.98 & 0.04 & 2.05 & 0.04 & 1.83 & 0.03 \\
\hline & $\mathbf{K}_{\mathrm{s}}$ & 91 & 0.05 & 46 & 0.07 & 47 & 0.07 & 62 & 0.10 & 66 & 0.08 & 90 & 0.11 \\
\hline & $\alpha$ & 0.58 & 0.06 & -0.08 & 0.08 & -0.09 & 0.17 & -0.29 & 0.09 & -0.24 & 0.05 & -0.21 & 0.42 \\
\hline & $\kappa$ & --- & --- & 0.24 & 0.03 & 0.27 & 0.02 & 0.52 & 0.06 & 0.42 & 0.03 & 0.33 & 0.09 \\
\hline$\overline{0}$ & $\beta$ & ---- & --- & --- & --- & --- & --- & 1.00 & 0.01 & 1.00 & 0.01 & 0.99 & 0.01 \\
\hline$\stackrel{0}{\Sigma}$ & $\xi$ & --- & --- & --- & --- & --- & --- & 1.03 & 0.03 & 1.04 & 0.03 & 0.95 & 0.02 \\
\hline ํํㄴ & $\phi_{1}$ & --- & --- & --- & --- & --- & --- & 0.98 & 0.00 & 0.95 & 0.00 & 0.94 & 0.00 \\
\hline 는 & $q_{0}$ & --- & --- & --- & --- & --- & --- & ---- & --- & --- & --- & 1.96 & 0.05 \\
\hline & $\gamma$ & ---- & ---- & --- & --- & ---- & ---- & ---- & ---- & ---- & ---- & -0.25 & 0.11 \\
\hline & $\tau$ & ---- & ---- & ---- & ---- & ---- & ---- & --- & --- & ---- & ---- & -0.38 & 0.29 \\
\hline
\end{tabular}

1074

1075

1076

1077 
Hydrol. Earth Syst. Sci. Discuss., doi:10.5194/hess-2017-9, 2017

Manuscript under review for journal Hydrol. Earth Syst. Sci.

Published: 17 January 2017

(c) Author(s) 2017. CC-BY 3.0 License.
Hydrology and (c)

Table 3. Maximum a posteriori (MAP) and coefficient of variation (CV) of inferred parameters, for all inferences with GR4J hydrological model. CVs equal or larger than 0.2 are marked in red colour. Inferred parameters by the TLs enforcement are marked in shadow.

1081

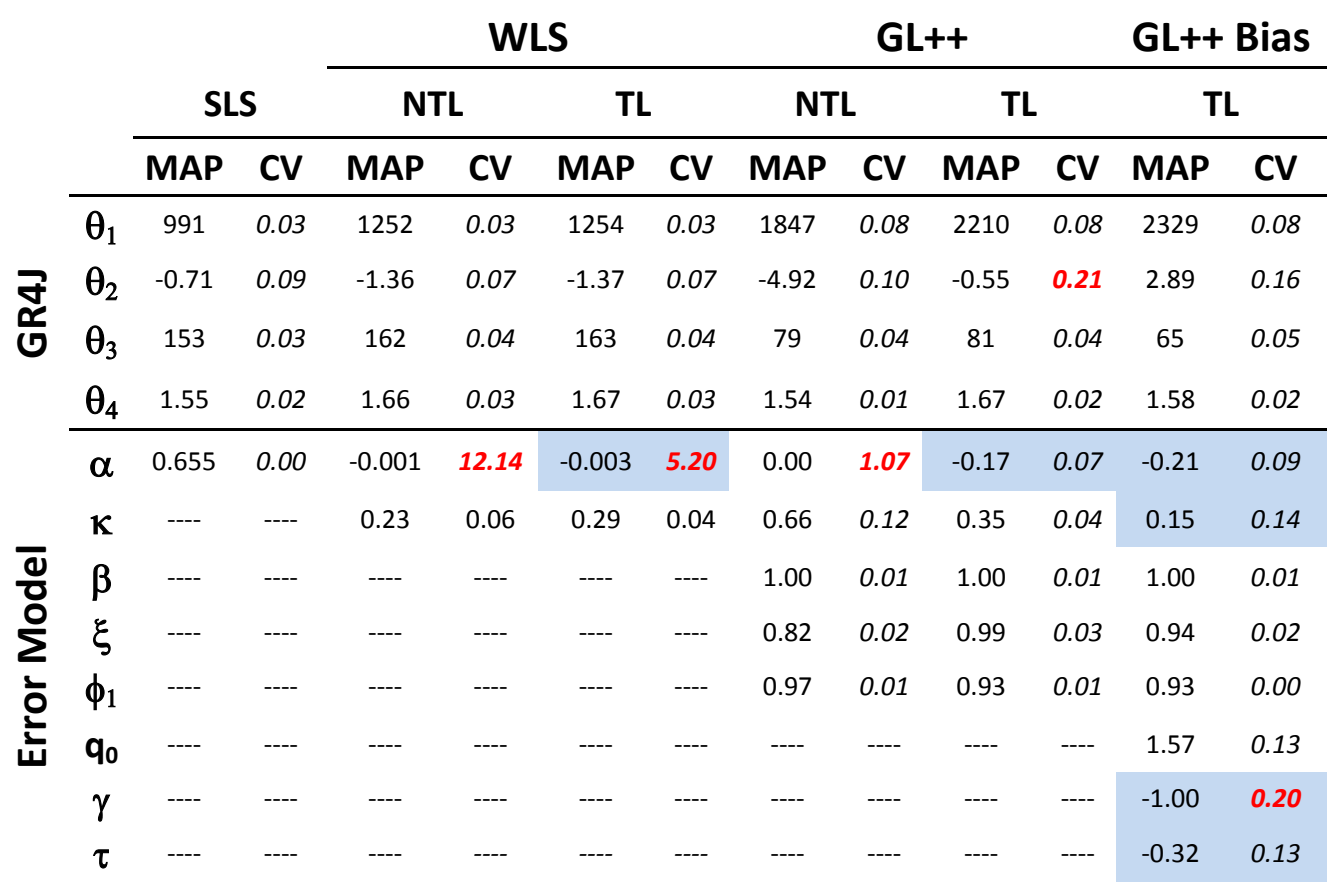

